\title{
Variational principles, completeness and the existence of traps in behavioral sciences
}

\author{
T. Q. Bao ${ }^{1}$ - S. Cobzaş ${ }^{2}$ - A. Soubeyran ${ }^{3}$
}

\begin{abstract}
In this paper, driven by Behavioral applications to human dynamics, we consider the characterization of completeness in pseudo-quasimetric spaces in term of a generalization of Ekeland's variational principle in such spaces, and provide examples illustrating significant improvements to some previously obtained results, even in complete metric spaces. At the behavioral level, we show that the completeness of a space is equivalent to the existence of traps, rather easy to reach (in a worthwhile way), but difficult (not worthwhile to) to leave. We first establish new forward and backward versions of Ekeland's variational principle for the class of strict-decreasingly forward (resp. backward)-lsc functions in pseudo-quasimetric spaces. We do not require that the space under consideration either be complete or to enjoy the limit uniqueness property since, in a pseudo-quasimetric space, the collections of forwardlimits and backward ones of a sequence, in general, are not singletons.
\end{abstract}

Keywords Pseudo-quasimetric · Forward-completeness · Backward-completeness · Variational principle $\cdot$ Group dynamics $\cdot$ Existence of trap

$凶 \quad$ T. Q. Bao

btruong@nmu.edu

S. Cobzaş

scobzas@math.ubbcluj.ro

A. Soubeyran

antoine.soubeyran@gmail.com

1 Department of Mathematics and Computer Science, Northern Michigan University, Marquette, MI, USA

2 Department of Mathematics, Babeş-Bolyai University, Cluj-Napoca, Romania

3 Aix-Marseille School of Economics, CNRS and EHESS, Aix-Marseille University, Marseille, France 


\section{Introduction}

This paper, driven by Behavioral applications to human dynamics, considers the mathematical completeness problem. We provide a characterization of completeness in pseudo-quasimetric spaces in terms of a generalization of the Ekeland theorem in such spaces. Notice that a pseudo-quasimetric is a distance which satisfies the triangular inequality, the distance from point " $y$ " to point " $x$ " can be different from the distance from " $x$ " to " $y$ ", the distance from a point to itself is zero, and it is possible that the distance from a point to a different one be zero. Our paper also provides examples illustrating significant improvements to some previously obtained results, even in complete metric spaces. Our motivations are twofold.

On one side, in Mathematics, a big issue is the COMPLETENESS PROBLEM, that is, to know when a space is complete. In such a case, a Cauchy sequence converges. In a recent survey Cobzaş (2016) presented various circumstances in which fixed point results and variational principles imply completeness. In this paper we will focus the attention on Ekeland's variational principle (Ekeland 1972), discovered by Ivar Ekeland in 1972. It is one of the most useful tools in nonlinear analysis and variational analysis. It allows us to study minimization problems in which the lower level set of the problem is not compact or, in other words, when the Bolzano-Weierstrass theorem can not be applied. More precisely, it asserts the existence of nearly optimal solutions to some optimization problems for the class of functions, defined on a complete metric space being lsc, bounded below, and proper (i.e. not identically equal to infinity). Our paper develops generalized versions of Ekeland's variational principle in pseudo-quasimetric spaces which are not necessarily complete.

On the other side, in Behavioral Sciences, a big issue is THE END PROBLEM, that is, to know when and where a human dynamic, which starts from an initial position, and follows a transition, defined as a succession of positions (which can be doings, havings or beings) ends somewhere.

Strikingly, this End problem in Behavioral Sciences is exactly the big Completeness problem in Mathematics. This comes from two simple remarks. In Behavioral Sciences, a Cauchy sequence refers to a dynamic (sequence) which agglomerates, but without knowing where, while a convergent dynamic refers to a sequence which approaches some end. The end problem refers to three fundamental questions in Behavioral Sciences:

(1) to know when an individual behavior is predictable (stabilizes) in order to solve coordination problems between interrelated agents in game theory;

(2) to modelize habituation processes (habit formation and breaking) where agents and organizations, progressively, perform more and more similar individual and collective actions in similar contexts, ending in habits and routines;

(3) to know when and where human dynamics end in traps, rather easy to reach, but difficult to leave, before being able to reach their desires.

A recent variational rationality approach (Soubeyran 2009, 2010, 2016 for a revised version) allows us to make the connection between these two big problems - the mathematical Completeness problem and the behavioral End problem. This Variational Rationality (abbreviated VR) approach proposes a way to model human behaviors as worthwhile approach or avoidance dynamics (see also Sect. 5, Conclusions, in this paper). Such dynamics start from some undesirable initial states, follow acceptable transitions (which are defined as successions of worthwhile stays and changes), and make attempts to approach and reach desired ends (desires), or to avoid the undesirable initial states. To be more concrete, stays refer to habits, routines, norms, rules, exploitation phases, etc. and changes refer to exploration, search, learning, innovation, etc. In such dynamics, at each step, if motivation to change is proportionally 
higher than or as high as resistance to change, it is worthwhile to change. If not, the dynamic process ends up in some variational trap which is worthwhile to approach and reach, but not worthwhile to leave. This new model of human behavior allowed to give a striking and surprising new interpretation of Ekeland's variational principle and of other famous variational principles in the particular case where motivation to change and resistance to change are identified to advantage and inconvenience to change, experience does not matter too much, and a pseudo-quasimetric models inconvenience to change rather than to stay as the difference between costs to be able to change and costs to be able to stay. In this VR approach, Ekeland's variational principle gives sufficient conditions for the existence of variational traps. In his formal proofs, Soubeyran $(2009,2010,2016)$ considered only the case of quasimetrics (in the sense used in the present paper, see Definition 2). Here, we extend the mathematical aspect to pseudo-quasimetrics where inconveniences to change rather than to stay can be zero, when costs to be able to change are equal to costs to be able to stay. We do even more. Sullivan (1981) showed that the original Ekeland's variational principle is equivalent to completeness of metric spaces. Karapinar and Romaguera (2015) proved that their weak version of Ekeland's variational principle is equivalent to completeness of quasimetric spaces. In this paper, we extend their results to the case of pseudo-quasimetric spaces. At the end of the last section, we show, as a direct application, that completeness of a pseudo-quasimetric space $X$ (the space of actions) is equivalent to the existence of variational traps (the VR meaning of Ekeland's variational principle), starting from any initial state. This is a very nice result in Behavioral Sciences: in a pseudo-quasimetric space, where the pseudo-quasimetric models an inconvenience to change, completeness of the space is equivalent to the fact that every worthwhile to change dynamic ends in some trap. Then, in such a complete space, no worthwhile dynamic can wander.

Ekeland's variational principle has been extended in many directions, without much justification, except for applications in Computer Science (see Aydi et al. 2015 and the references therein) and ones in Behavioral Sciences. For vector- and set-valued versions of Ekeland's variational principle, see Ansari (2007), Bao et al. (2015a,b,c). Since the vector- and setvalued versions are out of the scope of this paper, we focus our attention on the following issues.

- Topological spaces Ekeland's variational principles are still valid in quasimetric spaces (Al-Homidan et al. 2008; Bao 2015; Bao et al. 2015a, b, c, 2016; Cobzaş 2011; Karapinar and Romaguera 2015; Ume 2002) and partial-metric spaces (Aydi et al. 2015).

- Lower semicontinuity of cost functions Ekeland's variational principle could be established for decreasingly lsc functions (Bao 2015; Bao et al. 2015a,b, 2016; Kirk and Saliga 2001) and nearly lsc ones (Karapinar and Romaguera 2015).

- Limit uniqueness In the original Ekeland's variational principle this requirement is automatically satisfied since the limit (if exists) is unique in a metric space. This fact no longer holds true in (pseudo)-quasimetric spaces due to the lack of the symmetry axiom. A majority of publications in quasimetric spaces require that the space in question is complete and regular (Ume 2002), complete and $T_{1}$ (Cobzaş 2011), complete and Hausdorff (Bao et al. 2015a, b,c, 2016), or enjoys a less restrictive property: every generalizedPicard sequence has at most one forward-limit (Bao and Théra 2015, Theorem 4.5). In Karapinar and Romaguera (2015), formulated for the first time a weak version of this principle in complete and possibly not $T_{1}$ quasimetric spaces.

The core of the present paper is to start with a hypothesis about series completeness (see Definition 8). Roughly speaking, in terms of the VR approach of human behaviors (see Soubeyran 2016), we suppose that if the resources spend to move, following a succession (path) of stays and changes is finite (a natural hypothesis), then this human dynamic 
ends somewhere (converges). This gives a concrete meaning for the completeness assumption. More precisely, a sequence $\left\{x_{n}\right\}$ in a pseudo-quasimetric space $(X, q)$ is said to be forward-distance-series-convergent if the forward-distance series $\Sigma_{n=0}^{+\infty} q\left(x_{n}, x_{n+1}\right)<+\infty$, i.e., is convergent. Then, a pseudo-quasimetric space is said to be forward-distance-seriescomplete if every forward-distance-series-convergent sequence is forward-convergent. This assumption must be related to the definition of Kasahara spaces as $L$-spaces endowed with our series completeness assumption (Filip 2011, Definition 1.6.1).

The main purpose of this paper is to formulate new forward and backward versions of Ekeland's variational principle in possibly neither complete nor $T_{1}$ pseudo-quasimetric spaces. They reduce to the original principle when the space in question happens to be a complete metric space, while being able to be applied to more general settings when the existing results could not be used. Importantly, they give characterizations of the forward (resp. backward)completeness of pseudo-quasimetric spaces.

The paper is organized as follows. Section 2 presents basic definitions and preliminary results for pseudo-quasimetrics. In Sect. 3 we establish enhanced forward and backward versions of Ekeland's variational principle in pseudo-quasimetric spaces which may fail to be complete. We also add some comments on applications to Behavioral Sciences.

\section{Preliminaries}

We present and discuss definitions of generalized distances (known also as metrics) and of notions of closedness and completeness in topological spaces whose topologies are induced by these distances; (see, e.g., Bao and Théra 2015; Cobzaş 2011, 2013; Kelly 1963; Reilly and Subrahmanyam 1982; Wilson 1931a).

There are numerous ways of relaxing the axioms of a metric space, leading to various notions of generalized metric spaces. Let us start with some types of metrics.

\section{Definition 1 (types of metrics)}

(i) A METRIC on a nonempty set $X$ is a bifunction (called the distance function or simply distance) $d: X \times X \rightarrow \mathbb{R}$, where $\mathbb{R}$ is the set of real numbers, and for all $x, y, z \in X$ the following conditions are satisfied:

(d1) $d(x, y) \geq 0$ (nonnegativity axiom);

(d2) $d(x, y)=0$ if and only if $x=y$ (coincidence axiom);

(d3) $d(x, y)=d(y, x)$ (symmetry axiom);

(d4) $d(x, z) \leq d(x, y)+d(y, z)$ (subadditivity axiom).

The pair $(X, d)$ is called a metric space.

(ii) A PSEUDO- METRIC on $X$ is a bifunction $d: X \times X \rightarrow \mathbb{R}$ which satisfies the axioms for a metric, except that the coincidence axiom is replaced by the equality-implies-indistancy condition:

$\left(\mathrm{d} 2^{\prime}\right) d(x, x)=0$ for all $x \in X$ (but possibly $d(x, y)=0$ for $x \neq y$ ).

(iii) A QUASIMETRIC is defined by a bifunction that satisfies all the axioms of a metric with the possible exception of the symmetry axiom.

(iv) A PRE- METRIC is defined by a bifunction that satisfies conditions (d1) and (d2'). A SEMI- METRIC is a bifunction that satisfies conditions (d1), (d2) and (d3) (i.e. the triangle inequality is not satisfied), see Wilson (1931b).

In this paper we work on the following spaces. 
Definition 2 (pseudo-quasimetrics (Kelly 1963, Definition 2.1)). A PSEUDO- QUASIMETRIC $^{1}$ on a nonempty set $X$ is a bifunction $q: X \times X \rightarrow \mathbb{R}_{+}:=[0,+\infty)$, where for all $x, y, z \in X$ the following conditions are satisfied:

(q1) $x=y \Longrightarrow q(x, y)=0 \quad$ (equality implies indistancy);

(q2) $q(x, z) \leq q(x, y)+q(y, z) \quad$ (subadditivity, or triangle inequality).

Obviously, $q$ satisfies the nonnegativity axiom of a metric. As always, $(X, q)$ is called a PSEUDO- QUASIMETRIC SPACE.

If $q$ also satisfies the condition

(q3) $q(x, y)=0 \Longrightarrow x=y \quad$ (indistancy implies equality),

then $q$ is called a QUASIMETRIC.

Remark 1 In Cobzaş (2013) by a quasimetric one understands a bifunction $q: X \times X \rightarrow \mathbb{R}_{+}$ satisfying (q1), (q2) and

$\left(\mathrm{q}^{\prime}\right) q(x, y)=q(y, x)=0 \Longrightarrow x=y$.

This is partly justified by the fact that a pseudo-quasimetric $q$ satisfies (q3 $\left.3^{\prime}\right)$ if and only if the associated bifunction $q^{s}(x, y)=\max \{q(x, y), q(y, x)\}, x, y \in X$, is a metric on $X$. Notice that $(\mathrm{q} 1)+\left(\mathrm{q} 3^{\prime}\right) \nRightarrow(\mathrm{d} 2)$.

Example 1 (some important pseudo-quasimetrics) Let $X$ be a subset of $\mathbb{R}$ and define $q_{1}, q_{2}, q_{3}: X \times X \rightarrow \mathbb{R}_{+}$by

$$
\begin{gathered}
q_{1}(x, y)=\left\{\begin{array}{ll}
y-x & \text { if } y \geq x \\
0 & \text { otherwise }
\end{array}, \quad q_{2}(x, y)=\left\{\begin{array}{ll}
y-x & \text { if } y \geq x \\
1 & \text { otherwise }
\end{array},\right. \text { and }\right. \\
q_{3}(x, y)=\left\{\begin{array}{ll}
a(y-x) & \text { if } y \geq x \\
b(x-y) & \text { otherwise }
\end{array}, \text { where } a, b>0 .\right.
\end{gathered}
$$

- All of these bifunctions are pseudo-quasimetrics.

- $q_{2}$ and $q_{3}$ are quasimetrics, but $q_{1}$ is not since $q_{1}(2,1)=0$.

- The distance $q_{1}$ does not satisfy the reverse implication of (q1), but it satisfies ( $\left(3^{\prime}\right)$, i.e. it is a quasimetric space in the sense used in Cobzaş (2013).

Every pseudo-quasimetric space $(X, q)$ can be considered as a topological space. For $x \in X$ and $r>0$, we could define an open ball (resp. a closed ball) in $X$ by

$$
\mathbb{B}_{q}(x, r):=\{y \in X \mid q(x, y)<r\}\left(\text { resp. } \mathbb{B}_{q}[x, r]:=\{y \in X \mid q(x, y) \leq r\}\right) .
$$

The topology $\tau(q)$ of a pseudo-quasimetric space $(X, q)$ can be introduced by taking, for any $x \in X$, the collection $\left\{\mathbb{B}_{q}(x, r) \mid r>0\right\}$ as a base of the neighborhood filter of the point $x$.

Following Kelly (1963), consider a pseudo-quasimetric $q$ over a nonempty set $X$. The conjugate of $q$, denoted by $\bar{q}: X \times X \rightarrow \mathbb{R}_{+}$and defined by $\bar{q}(x, y)=q(y, x), \forall x, y \in X$, is a pseudo-quasimetric as well, generating a topology $\tau(\bar{q})$. As a space equipped with two

1 In Bao (2015), Bao et al. (2015a, b, c, 2016), Bao and Théra (2015) and the references therein, by a pseudoquasimetric space one understands a quasimetric space. 
topologies, $\tau(q)$ and $\tau(\bar{q})$, the triple $(X, q, \bar{q})$ is a bitopological space. ${ }^{2}$ The bifunction $q^{s}(x, y)=\max \{q(x, y), \bar{q}(x, y)\}, x, y \in X$, is a pseudo-metric which is a metric if and only if $q$ satisfies condition ( $\mathrm{q}^{\prime}$ ) from Remark 1 .

Let us describe the open balls in $\mathbb{R}$ with respect to the pseudo-quasimetrics in Example 1.

$$
\begin{aligned}
& \mathbb{B}_{q_{1}}(x, r)=(-\infty, x+r), \quad \mathbb{B}_{\bar{q}_{1}}(x, r)=(x-r,+\infty), \\
& \mathbb{B}_{q_{2}}(x, r)=[x, x+r) \text { for all } r \in(0,1) \text { and }(-\infty, x+r) \text { for all } r \in[1,+\infty), \\
& \mathbb{B}_{\bar{q}_{2}}(x, r)=(x-r, x] \text { for all } r \in(0,1) \text { and }(x+r,+\infty) \text { for all } r \in[1,+\infty), \\
& \mathbb{B}_{q_{3}}(x, r)=(x-b r, x+a r), \mathbb{B}_{\bar{q}_{3}}(x, r)=(x-a r, x+b r) .
\end{aligned}
$$

Notice that the quasimetric $q_{3}$ satisfies

$$
\min \{a, b\} \cdot|y-x| \leq q_{3}(x, y) \leq \max \{a, b\} \cdot|y-x| \text { for all } x, y \in \mathbb{R},
$$

so that the topology defined by $q_{3}$ agrees with the usual topology of $\mathbb{R}$.

In contrast to metric spaces, in bitopological pseudo-quasimetric spaces there are two different notions of convergence (with respect to each topology). ${ }^{3}$

Definition 3 (convergence in pseudo-quasimetric spaces, (Bao and Théra 2015, Definition 4$)$ ). Let $\left\{x_{n}\right\}$ be a sequence in a bitopological pseudo-quasimetric $(X, q, \bar{q})$. We say that:

(i) the sequence $\left\{x_{n}\right\}$ is FORWARD- CONVERGENT to $x_{*}$, if it converges to $x_{*}$ with respect to $\tau(\bar{q})$, i.e., $\bar{q}\left(x_{*}, x_{n}\right)=q\left(x_{n}, x_{*}\right) \rightarrow 0$.

(ii) the sequence $\left\{x_{n}\right\}$ is BACKWARD CONVERGENT to $x_{*}$, if it converges to $x_{*}$ with respect to the topology $\tau(q)$, i.e., $q\left(x_{*}, x_{n}\right) \rightarrow 0$;

We use the following notation:

(iii) $\overrightarrow{\left\{x_{n}\right\}}$ stands for the collection of all forward-limits of the sequence $\left\{x_{n}\right\}$ and thus $\overrightarrow{\{x\}}=\{y \in X \mid q(x, y)=0\}=\overline{\{x\}}^{\bar{q}}$ is the set of all forward-limits of the constant sequence $x_{n}=x$ for all $n \in \mathbb{N}$, where the upper index $\bar{q}$ mentions the topology $\tau(\bar{q})$ of convergence;

(iv) $\overleftarrow{\left\{x_{n}\right\}}$ stands for the collection of all backward limits of the sequence $\left\{x_{n}\right\}$ and thus $\overleftarrow{\{x\}}=\{y \in X \mid q(y, x)=0\}=: \overline{\{x\}}^{q}$, where the upper index $q$ mentions the topology $\tau(q)$ of convergence.

Due to the lack of the symmetry axiom in pseudo-quasimetric spaces, the definition of Cauchy sequences could be generalized in many ways.

Definition 4 (Cauchy sequences in pseudo-quasimetrics (Bao and Théra 2015, Definition 5)). Let $\left\{x_{n}\right\}$ be a sequence in a pseudo-quasimetric space $(X, q)$. We say that:

(i) the sequence $\left\{x_{n}\right\}$ is FORWARD- CAUCHY ${ }^{4}$, if for each $\varepsilon>0$ there is an integer $N_{\varepsilon} \in \mathbb{N}$ such that $q\left(x_{n}, x_{n+k}\right)<\varepsilon$ for all $n \geq N_{\varepsilon}$ and $k \in \mathbb{N}$;

\footnotetext{
2 Kelly (1963, p. 71) "The notion of a bitopological space used in relation to semi-continuous functions restores sufficient symmetry to enable one to use some of the existing techniques of continuous functions."

3 It has been also defined a sequence as being bi-convergent if it is both forward- and backward-convergent, (i.e. if it convergent with respect to the pseudo-metric $q^{s}$ ).

4 Known also as left-Cauchy (Bao et al. 2015a,b,c), q-Cauchy Kelly (1963), and left $K$-Cauchy (Reilly and Subrahmanyam 1982)
} 
(ii) the sequence $\left\{x_{n}\right\}$ is BACKWARD CAUCHY ${ }^{5}$, if for each $\varepsilon>0$ there is an integer $N_{\varepsilon} \in \mathbb{N}$ such that $q\left(x_{n+k}, x_{n}\right)<\varepsilon$ for all for all $n \geq N_{\varepsilon}$ and $k \in \mathbb{N}$;

(iii) the sequence $\left\{x_{n}\right\}$ is CAUCHY ${ }^{6}$, if it is both forward- and backward-Cauchy, i.e., for each $\varepsilon>0$ there is an integer $N_{\varepsilon} \in \mathbb{N}$ such that $q\left(x_{m}, x_{n}\right)<\varepsilon$ for all $m, n \geq N_{\varepsilon}$. This is equivalent to the fact that it is Cauchy with respect to the associated pseudo-metric $q^{s}$.

Definition 5 (completeness in pseudo-quasimetric spaces) Let $(X, q)$ be a pseudoquasimetric space. We say:

(i) the space is FORWARD- FORWARD- COMPLETE (resp. backward-backward-complete), if every forward- (resp. backward-)Cauchy sequence is forward- (resp. backward-) convergent to some forward- (resp. backward-)limit;

(ii) the space is FORWARD- BACKWARD- COMPLETE (resp. backward-forward-complete), if every forward- (resp. backward-)Cauchy sequence is backward- (resp. forward-) convergent to some backward- (resp. forward-)limit;

(iii) the space is FORWARD- COMPLETE (resp. BACKWARD- COMPLETE), if every Cauchy sequence is forward- (resp. backward-)convergent to some forward- (resp. backward-) limit.

With two notions of convergence and three kinds of Cauchy sequences defined in a pseudoquasimetric space, we could define six types of completeness. The reader is referred to Reilly and Subrahmanyam (1982, Examples 2-3) for differences between various Cauchy and completeness notions.

Remark 2 The notions with forward and backward used here differ from the notions of left and right used in Cobzaş (2013). For convenience we present the equivalences between the corresponding notions. For a sequence $\left\{x_{n}\right\}$ in a pseudo-quasimetric space $(X, q)$ :

- forward-Cauchy means left $q$ - $K$-Cauchy, or equivalently, right $\bar{q}$ - $K$-Cauchy.

- backward Cauchy means right $q$ - $K$-Cauchy or, equivalently, left $\bar{q}$ - $K$-Cauchy.

For the space $X$ :

- forward-forward-complete means right $\bar{q}-K$-complete;

- backward-backward-complete means right $q$ - $K$-complete;

- backward-forward-complete means left $\bar{q}-K$-complete;

- forward-backward-complete means left $q$ - $K$-complete.

Remark 3 (on 'left' terminologies)

When calling the ball $\mathbb{B}(x, r)$ a left ball (Cobzaş 2011, 2013), it would be logic to say that the topology $\tau(q)$ is a left topology, and a sequence $\left\{x_{n}\right\}$ converging to $x_{*}$ with respect to the topology $\tau(q)$ is left-convergent. Observe that doing this would lead to some contradictions between known 'left' concepts in the literature.

Assume, in addition, that the forward distance series is convergent, i.e.,

$$
\sum_{n=1}^{\infty} q\left(x_{n}, x_{n+1}\right)<+\infty .
$$

It is easy to check (see, e.g., Bao and Théra 2015, Proposition 2.2) that the sequence is forward-Cauchy, or left-Cauchy. It is natural to say that the space is left-complete if every

\footnotetext{
5 Known also as $\bar{q}$-Cauchy (Kelly 1963), and right $K$-Cauchy (Reilly and Subrahmanyam 1982)

6 Known also as bi-Cauchy, or Cauchy in two topologies
} 
left-Cauchy sequence is left-convergent. Doing so, the sequence under consideration is leftconvergent to some $x_{*}$, i.e., $q\left(x_{*}, x_{n}\right) \rightarrow 0$ as $n \rightarrow \infty$. Unfortunately, it conflicts with the so-called left-convergence in Bao et al. $(2015 \mathrm{a}, \mathrm{b}, \mathrm{c})$ and earlier publications mentioned therein.

Another reason for not mentioning the topologies in the definitions of convergence and Cauchyness is that a backward-convergent sequence is related to the topology $\tau(q)$ $\left(\lim _{n \rightarrow \infty} q\left(x_{*}, x_{n}\right)=0 \Longleftrightarrow \forall \varepsilon>0, \exists N_{\varepsilon} \in \mathbb{N}: \forall n \geq N_{\varepsilon}, x_{n} \in \mathbb{B}_{q}(x, \varepsilon)\right)$, while a backward-Cauchy sequence is related to the topology $\tau(\bar{q})$ ( $\left\{x_{n}\right\}$ is backward-Cauchy $\left.\Longleftrightarrow \forall \varepsilon>0, \exists N_{\varepsilon} \in \mathbb{N}: \forall k \in \mathbb{N}, \forall n \geq N_{\varepsilon}, x_{n+k} \in \mathbb{B}_{\bar{q}}\left(x_{n}, \varepsilon\right)\right)$.

Wilson (1931a) proved that if $\left\{x_{n}\right\}$ is both forward- and backward-convergent to $x_{*}$ in a quasimetric space $(X, q)$, then $x_{*}$ is the only limit point of $\left\{x_{n}\right\}$ of any kind and $\left\{x_{n}\right\}$ is Cauchy. He also showed that if $\left\{x_{n}\right\}$ has more than one forward- (backward-)limit point then $\left\{x_{n}\right\}$ has no backward- (forward-)limit point. Notice that Wilson used the terms of u-limit and 1-limit (from 'upper' and 'lower') instead.

Theorem 1 ( (Wilson 1931a, Theorems I and II), see also (Cobzaş 2013, Proposition 1.2.4)) Let $\left\{x_{n}\right\}$ be a sequence in a pseudo-quasimetric space $(X, q)$.

(1) If $\left\{x_{n}\right\}$ is forward-convergent to $x_{*}$ and backward-convergent to $x^{*}$, then $q\left(x^{*}, x_{*}\right)=0$.

(2) If $\left\{x_{n}\right\}$ is forward-convergent to $x_{*}$ and $q\left(x_{*}, y_{*}\right)=0$, then $\left\{x_{n}\right\}$ is forward-convergent to $y_{*}$.

(3) If a forward-Cauchy sequence $\left\{x_{n}\right\}$ has a subsequence which is forward(backward-)convergent to $x_{*}$ (to $\left.x^{*}\right)$, then $\left\{x_{n}\right\}$ is forward-(backward-) convergent to $x_{*}$ (to $\left.x^{*}\right)$.

Proof For reader's convenience we include the simple proofs.

(1) If $q\left(x_{n}, x_{*}\right) \rightarrow 0$ and $q\left(x^{*}, x_{n}\right) \rightarrow 0$, then

$$
q\left(x^{*}, x_{*}\right) \leq q\left(x^{*}, x_{n}\right)+q\left(x_{n}, x_{*}\right) \rightarrow 0 \text { as } n \rightarrow \infty .
$$

(2) If $q\left(x_{n}, x_{*}\right) \rightarrow 0$ and $q\left(x_{*}, y_{*}\right)=0$, then

$$
q\left(x_{n}, y_{*}\right) \leq q\left(x_{n}, x_{*}\right)+q\left(x_{*}, y_{*}\right)=q\left(x_{n}, x_{*}\right) \rightarrow 0 \text { as } n \rightarrow \infty .
$$

(3) Suppose that $\left\{x_{n}\right\}$ is a forward-Cauchy sequence having a subsequence $\left\{x_{n_{k}}\right\}$ forwardconvergent to some $x_{*} \in X$, that is $\lim _{k} q\left(x_{n_{k}}, x_{*}\right)=0$.

For $\varepsilon>0$ let $k_{0}, n_{0} \in \mathbb{N}$ be such that

$$
q\left(x_{n_{k}}, x_{*}\right)<\varepsilon, \forall k \geq k_{0}, \text { and } q\left(x_{n}, x_{m}\right)<\varepsilon, \forall m, n \text { with } n_{0} \leq n<m .
$$

For $n \geq n_{0}$ let $k \geq k_{0}$ be such that $n_{k}>n$. Then

$$
q\left(x_{n}, x_{*}\right) \leq q\left(x_{n}, x_{n_{k}}\right)+q\left(x_{n_{k}}, x_{*}\right)<2 \varepsilon .
$$

Suppose now the forward-Cauchy sequence $\left\{x_{n}\right\}$ has a subsequence $\left\{x_{n_{k}}\right\}$ backwardconvergent to $x^{*} \in X$, that is $\lim _{k} q\left(x^{*}, x_{n_{k}}\right)=0$. For $\varepsilon>0$ let $n_{0} \in \mathbb{N}$ be such that $q\left(x_{n}, x_{m}\right)<\varepsilon, \forall m, n$ with $n_{0} \leq n<m$ and let $k_{0} \in \mathbb{N}$ be such that $n_{k_{0}} \geq n_{0}$ and $q\left(x^{*}, x_{n_{k}}\right)<\varepsilon, \forall k \geq k_{0}$. Then for $n \geq n_{k_{0}}, q\left(x^{*}, x_{n}\right) \leq q\left(x^{*}, x_{n_{k_{0}}}\right)+q\left(x_{n_{k_{0}}}, x_{n}\right)<2 \varepsilon$.

We present now the separation axioms in topological spaces. 
Definition 6 A topological space $(X, \tau)$ is:

(i) $T_{0}$ if for every pair of distinct points $x, y \in X$, at least one of them has a neighborhood which does not contain the other;

(ii) $T_{1}$ if for every pair of distinct points $x, y \in X$, there exist the neighborhoods $U$ of $x$ and $V$ of $y$ such that $y \notin U$ and $x \notin V$;

(iii) $T_{2}$ (or HAUSDORFF) if for every pair of distinct points $x, y \in X$, there exist the neighborhoods $U$ of $x$ and $V$ of $y$ such that $U \cap V=\emptyset$;

(iv) REGULAR if for every point $x \in X$ and closed set $A$ not containing $x$ there exist the disjoint open sets $U, V$ such that $x \in U$ and $A \subset V$; the space $X$ is called $T_{3}$ if it is $T_{1}$ and regular;

(v) NORMAL if for every pair of disjoint closed subsets $A, B$ of $X$ there exist the disjoint open sets $U, V$ such that $A \subset U$ and $B \subset V$; the space $X$ is called $T_{4}$ if it is $T_{1}$ and normal.

Notice that some authors include in the definition of regular and normal spaces the separation condition $T_{1}$.

Remark 4 (a) A topological space $(X, \tau)$ is $T_{0}$ if and only if $x \in \overline{\{y\}}^{\tau}$ and $y \in \overline{\{x\}}^{\tau}$ implies $x=y$. One defines an order on a $T_{0}$ topological space by $x \leq_{\tau} \quad y$ if $x \in \overline{\{y\}}^{\tau}$. This combination of order and topology in $T_{0}$ spaces found applications in theoretical computer science, see Gierz et al. (2003) for a comprehensive presentation and GoubaultLarrecq (2013) for a good introduction to the area.

(b) A topological space $(X, \tau)$ is $T_{1}$ if and only if $\overline{\{x\}}^{\tau}=\{x\}$, for all $x \in X$, i.e. the one-point sets are closed.

We present some topological properties of pseudo-quasimetric spaces.

Theorem 2 (Cobzaş 2013). Let $(X, q)$ be a pseudo-quasimetric space. Then, the following are true:

(1) The ball $\mathbb{B}_{q}(x, r)$ is open in the topology $\tau(q)$ and the ball $\mathbb{B}_{q}[x, r]$ is closed in the topology $\tau(\bar{q})$. The ball $\mathbb{B}_{q}[x, r]$ need not be closed in the topology $\tau(q)$.

(2) For every fixed element $x \in X$, the function $q(x, \cdot):(X, q) \rightarrow(\mathbb{R},|\cdot|)$ is usc with respect to the topology $\tau(q)$ and lsc with respect to the topology $\tau(\bar{q})$.

(3) For every fixed element $y \in X$, the mapping $q(\cdot, y):(X, q) \rightarrow(\mathbb{R},|\cdot|)$ is lsc with respect to the topology $\tau(q)$ and usc with respect to the topology $\tau(\bar{q})$.

(4) If $q$ is a quasimetric, then the topology $\tau(q)$ is $T_{0}$, but not necessarily $T_{1}{ }^{7}$

(5) If the function $q(x, \cdot):(X, q) \rightarrow(\mathbb{R},|\cdot|)$ is continuous with respect to the topology $\tau(q)$ for every $x \in X$, then the topology $\tau(q)$ is regular.

(6) If $q(x, \cdot)$ is continuous with respect to the topology $\tau(\bar{q})$ for every $x \in X$, then the topology $\tau(\bar{q})$ is semi-metrizable.

We conclude this section with an important observation clearly implying that the results obtained in Bao (2015), Bao et al. (2015a,b,c, 2016), Bao and Théra (2015) are indeed established for quasimetric spaces in the sense of Definition 2.

Proposition 1 Let $(X, q)$ be a pseudo-quasimetric space.

(1) The space $(X, \tau(q))$ is $T_{1}$ if and only if $q(x, y)>0$ for every pair $x, y$ of distinct points in $X$, i.e. if $q$ is a quasimetric. In this case $(X, \tau(\bar{q}))$ is $T_{1}$ as well.

7 Recall that in Cobzaş (2013) a quasimetric satisfies the condition: $q(x, y)=q(y, x)=0$ implies $x=y$. 
(2) The space $(X, \tau(\bar{q}))((X, \tau(q)))$ is $T_{2}$ if and only if every sequence in $X$ has at most one forward-(backward-)limit.

Proof (1) Suppose that $q$ is a quasimetric. If $x, y \in X$ are distinct, then $r:=q(x, y)>0$ and $r^{\prime}:=q(y, x)>0$. It follows that $U=\mathbb{B}_{q}(x, r)$ is a $\tau(q)$-neighborhood of $x$ with $y \notin U$, and $V=\mathbb{B}_{q}\left(y, r^{\prime}\right)$ is a $\tau(q)$-neighborhood of $y$ with $x \notin V$.

Conversely, if there exists a pair $x, y$ of distinct points with $q(x, y)=0$, then $y \in \mathbb{B}(x, \varepsilon)$ for every $\varepsilon>0$, showing that the topology $\tau(q)$ is not $T_{1}$. Similarly, $\bar{q}(y, x)=q(x, y)=$ $0<\varepsilon$ implies $x \in \mathbb{B}_{\bar{q}}(y, \varepsilon)$ for all $\varepsilon>0$, showing that the topology $\tau(\bar{q})$ is not $T_{1}$.

(2) Suppose that $(X, \tau(\bar{q}))$ is $T_{2}$ and let $\left\{x_{n}\right\}$ be forward-convergent to $x_{*}$. If $y_{*} \neq x_{*}$, then there exists $r>0$ such that $\mathbb{B}_{\bar{q}}(x, r) \cap \mathbb{B} \bar{q}(y, r)=\emptyset$. By hypothesis, there exists $n_{0} \in \mathbb{N}$ such that $x_{n} \in \mathbb{B}_{\bar{q}}\left(x_{*}, r\right)$ for all $n \geq n_{0}$, implying $x_{n} \notin \mathbb{B}_{\bar{q}}\left(y_{*}, r\right)$, for all $n \geq n_{0}$, that is $\left\{x_{n}\right\}$ is not forward-convergent to $y_{*}$.

Suppose now that $(X, \tau(\bar{q}))$ is not $T_{2}$. Then there exist two points $x_{*} \neq y_{*}$ in $X$ such that $\mathbb{B}_{\bar{q}}\left(x_{*}, 1 / n\right) \cap \mathbb{B}_{\bar{q}}\left(y_{*}, 1 / n\right)=\emptyset$ for all $n \in \mathbb{N}$. Choosing an $x_{n}$ in this intersection, it follows that $q\left(x_{n}, x_{*}\right)<1 / n$ and $q\left(x_{n}, y_{*}\right)<1 / n$, for all $n$, which shows that the sequence $\left\{x_{n}\right\}$ is forward-convergent to both $x_{*}$ and $y_{*}$.

Example 2 In a case of a pseudo-metric space $(X, d)$ the condition $d(x, y)>0$ for all distinct $x, y$ implies that the space $X$ is normal, even $T_{4}$. The situation is different in the quasimetric case. We present an example of a quasimetric space which is not Hausdorff (i.e. $T_{2}$ ).

Let $X=\left\{x_{n}: n \in \mathbb{N}\right\} \cup\left\{x_{*}, y_{*}\right\}$ where all the points are pairwise distinct. Define

$$
\begin{aligned}
& q(u, u)=0, \forall u \in X, \quad q\left(x_{n}, x_{m}\right)=1, \forall m, n \in \mathbb{N}, m \neq n, \\
& q\left(x_{n}, x_{*}\right)=q\left(x_{n}, y_{*}\right)=1 / n \text { and } q\left(x_{*}, x_{n}\right)=q\left(y_{*}, x_{n}\right)=1, \forall n \in \mathbb{N}, \\
& q\left(x_{*}, y_{*}\right)=q\left(y_{*}, x_{*}\right)=1 .
\end{aligned}
$$

It is easy to check that $q$ is a quasimetric. The sequence $\left\{x_{n}\right\}$ is forward-convergent to $x_{*}$ and to $y_{*}$, so that the topology $\tau(q)$ is not Hausdorff, but it is obviously $T_{1}$, as $q(u, v)>0$ for all $u, v \in X, u \neq v$.

Incidentally, this gives also an example of a forward-convergent sequence which is not forward-Cauchy.

Remark 5 A deep result in functional analysis asserts that if a topological vector space $X$ is $T_{0}$, then it is $T_{1}$ and regular (i.e. $T_{3}$ ), see (Megginson 1998, Theorem 2.2.14). If $X$ is a finite dimensional asymmetric locally convex (in particular, asymmetric normed) space satisfying $T_{1}$, then it is topologically and algebraically isomorphic to $\mathbb{R}^{m}$, where $m \in \mathbb{N}$ is the algebraic dimension of $X$ (see (Cobzaş 2013, Proposition 1.1.68)

We don't know whether there exists or not an infinite dimensional asymmetric normed space which is $T_{1}$ but not $T_{2}$. For a characterization of Hausdorff property in asymmetric locally convex (in particular, in asymmetric normed) spaces, see (Cobzaş 2013, Proposition 1.1.63).

\section{Variational principles in pseudo-quasimetric spaces}

Ekeland's variational principle, first discovered by Ekeland (1972), is one of the most useful tools in nonlinear analysis and variational analysis. It can be used when the lower level set of a minimization problems is not compact. It leads to a quick proof of Caristi's fixed point 
theorem and it has been shown by Sullivan (1981) to be equivalent to the completeness of the corresponding metric space.

Theorem 3 (Ekeland and Turnbull 1983; cf. Ekeland 1972) Let $\varphi: X \rightarrow \mathbb{R} \cup\{+\infty\}$ be an extended real-valued function on a complete metric space $(X, d)$ which is $l s c$, bounded below, and not identically equal to $+\infty$. For any $\varepsilon>0$ consider $x_{0} \in \operatorname{dom} \varphi$ satisfying $\varphi\left(x_{0}\right) \leq \inf _{x \in X} \varphi(x)+\varepsilon$, i.e., $x_{0}$ is an $\varepsilon$-minimal solution of $\varphi$. Then for each $\lambda>0$, there exists a point $x_{*} \in \operatorname{dom} \varphi$ such that

$$
\begin{aligned}
& \text { (i) } \varphi\left(x_{*}\right) \leq \varphi\left(x_{0}\right) \\
& \text { (ii) } d\left(x_{0}, x_{*}\right) \leq \lambda \\
& \text { (iii) } \varphi(x)+(\varepsilon / \lambda) d\left(x_{*}, x\right)>\varphi\left(x_{*}\right), \forall x \in X \backslash\left\{x_{*}\right\} .
\end{aligned}
$$

If for $\gamma>0$ the set-valued mapping $S_{\gamma, d}: X \rightrightarrows X$ is defined by

$$
S_{\gamma, d}(x):=\{y \in X \mid \gamma d(x, y) \leq \varphi(x)-\varphi(y)\}, \forall x \in X,
$$

then the conclusions (i) and (ii) of Theorem 3 can be expressed in the form: there is $x^{*} \in$ $S_{\varepsilon / \lambda, d}\left(x_{0}\right)$ such that $S_{\varepsilon / \lambda, d}\left(x_{*}\right)=\left\{x_{*}\right\}$.

Recently, this principle has been extended to the class of pseudo-quasimetric spaces in which the symmetry axiom of the metric $d$ is dropped and the coincidence one is weaken to the equality-implies-indistancy condition, i.e., $d(x, x)=0$ for all $x \in X$. It is important to emphasize that under the hypothesis made in a majority of extensions, the pseudo-quasimetric space is indeed a quasimetric one; see Proposition 1. We pay our attention to the following developments:

- In Ume (2002, Theorem 2.9), it is assumed that the space $(X, q)$ is a forward-forwardcomplete $^{8}$ quasimetric space and that the function $q(x, \cdot): X \rightarrow \mathbb{R}$ is lsc. Note that it is not clear in the paper whether the function is lsc with respect to the topology $\tau(q)$ or the topology $\tau(\bar{q})$. As it is remarked in Cobzaş (2011, Remark 1.4), if $q(x, \cdot)$ is lsc with respect to the topology $\tau(q)$, then the topology $\tau(q)$ is regular.

- In Cobzaş (2011, Theorem 2.4), the space $(X, q)$ in question is assumed to be a $T_{1}$ quasimetric $^{9}$ space being either forward-forward-complete or backward-backwardcomplete ${ }^{10}$. Karapinar and Romaguera (2015) further extended Cobzaş' result to a weak form of Ekeland's variational principle in (not necessarily $T_{1}$ ) pseudo-quasimetric spaces, yielding a characterization of backward-backward completeness of these spaces.

- In Bao (2015), Bao et al. (2015a, b, c) worked with forward-forward-complete quasimetric spaces satisfying the forward Hausdorff property. ${ }^{11}$

Remark 6 Unfortunately, these results can not be applied to the pseudo-quasimetric space $\left(\mathbb{R}, q_{1}\right)$, where $q_{1}$ is defined in Example 1 , since the topology induced by $q_{1}$ is not $T_{1}$ and since it is not forward-forward-complete. The incompleteness of this space is obvious since the forward-Cauchy sequence $\left\{x_{n}\right\}$ with $x_{n}=-n$ has no forward-limit. It is a forward-Cauchy sequence since $q\left(x_{n}, x_{n+k}\right)=q(-n,-n-k)=0$ for all $n, k \in \mathbb{N}$. Fix an arbitrary number

\footnotetext{
8 Left-complete

9 A quasimetric in the sense of Cobzaş (2011), Cobzaş is a pseuso-quasimetric satisfying $q(x, y)=q(y, x)=$ $0 \Longrightarrow x=y$.

10 There are two versions of Ekeland's variational principle with respect to each kind of completeness.

11 A quasimetric space enjoys the forward Hausdorff property, i.e. a sequence being forward-convergent has a unique forward-limit.
} 
$x_{*} \in \mathbb{R}$, then $x_{*}>x_{n}$ for all sufficiently large $n \in \mathbb{N}$, and thus $q\left(x_{n}, x_{*}\right)=x_{*}+n \rightarrow+\infty$ as $n \rightarrow \infty$. Since $x_{*}$ was arbitrary, the sequence $\left\{x_{n}\right\}$ has no forward-limit.

In fact the following result holds in $\left(\mathbb{R}, q_{1}\right)$. For a sequence $\left\{x_{n}\right\}$ in $\mathbb{R}$

$$
\overrightarrow{\left\{x_{n}\right\}}= \begin{cases}(-\infty, a] & \text { if } a \in \mathbb{R}, \\ \mathbb{R} & \text { if } a=+\infty, \\ \emptyset & \text { if } a=-\infty,\end{cases}
$$

where $\overrightarrow{\left\{x_{n}\right\}}$ denotes the set of the forward-limits and $a=\liminf _{n \rightarrow \infty} x_{n}$.

The following examples partly motivate us to accomplish a study of Ekeland's variational principles in (not necessarily $T_{1}$ ) pseudo-quasimetric spaces.

Example 3 Consider a function $\varphi:\left(\mathbb{R}, q_{1}\right) \rightarrow(\mathbb{R},|\cdot|)$ defined by $\varphi(x)=e^{x}$. Obviously, it is bounded from below by 0 and $S_{1, q_{1}}(x)=(-\infty, x]$ for all $x \in X$, where $S_{1, q_{1}}$ is defined in (3.1). Therefore, there is no element $x_{*} \in \mathbb{R}$ such that $S_{1, q_{1}}\left(x_{*}\right)=\left\{x_{*}\right\}$ and the conclusion of Ekeland's variational principle does not hold true for this function $\varphi$ in the pseudo-quasimetric space $\left(\mathbb{R}, q_{1}\right)$.

Example 4 Consider a function $\varphi:\left([-1,1], q_{1}\right) \rightarrow(\mathbb{R},|\cdot|)$ defined by $\varphi(x)=e^{x}$. Obviously, it is bounded from below by 0 and $S_{1, q_{1}}(x)=[-1, x]$ for all $x \in[-1,1]$. Therefore, $x_{*}=-1$ satisfies the conclusion of Ekeland's variational principle for any starting point $x_{0} \in[-1,1]$.

Example 5 Consider a function $\varphi:\left(\mathbb{R}, \bar{q}_{1}\right) \rightarrow(\mathbb{R},|\cdot|)$ defined by $\varphi(x)=e^{x}$. It is easy to check that $S_{\varphi, 1}(1)=[0,1]$ and $S_{\varphi, 1}(0)=\{0\}$ showing that Ekeland's variational principle holds with the desired point $x_{*}=0$.

A question arises: under what requirements does Ekeland's variational principle hold in pseudo-quasimetric spaces? This question is answered in this section. We also prove that our new versions are characterizations of completeness of pseudo-quasimetric spaces.

Proposition 2 Let $(X, q)$ be a pseudo-quasimetric space and $\varphi: X \rightarrow \mathbb{R} \cup\{+\infty\}$ be a proper function (i.e., with $\operatorname{dom} \varphi \neq \emptyset$ ). Given $\lambda>0$, associate with $\varphi$ and $q$ the set-valued mapping $S_{\lambda, q}: X \rightrightarrows X$ defined by

$$
S_{\lambda, q}(x)=\{y \in X \mid \varphi(y)+\lambda q(x, y) \leq \varphi(x)\}, \quad \forall x \in X .
$$

If $x \in X \backslash \operatorname{dom} \varphi$ (i.e. if $\varphi(x)=+\infty)$, then $S_{\lambda, q}(x)=X$. For $x \in \operatorname{dom} \varphi, S_{\lambda, q}$ can be written as

$$
S_{\lambda, q}(x)=\{y \in X \mid \lambda q(x, y) \leq \varphi(x)-\varphi(y)\}, \quad \forall x \in X .
$$

Then $S_{\lambda, q}$ enjoys the following properties:

(1) $x \in S_{\lambda, q}(x)$ for all $x \in \operatorname{dom} \varphi$;

(2) if $x \in \operatorname{dom} \varphi$ and $y \in S_{\lambda, q}(x)$, then $\varphi(y) \leq \varphi(x)$ and $S_{\lambda, q}(y) \subset S_{\lambda, q}(x)$.

Proof The relation from (1) follows by the definition of $S_{\lambda, q}$. (2) Since $y \in S_{\lambda, q}(x) \Longleftrightarrow$ $\lambda q(x, y) \leq \varphi(x)-\varphi(y)$, it follows $\varphi(y) \leq \varphi(x)$ for $y \in S_{\lambda, q}(x)$. Also, $z \in S_{\lambda, q}(y) \Longleftrightarrow$ $\lambda q(y, z) \leq \varphi(y)-\varphi(z)$ and

$$
\lambda q(x, z) \leq \lambda q(x, y)+\lambda q(y, z) \leq \varphi(x)-\varphi(y)+\varphi(y)-\varphi(z)=\varphi(x)-\varphi(z),
$$

implies $z \in S_{\lambda, q}(x)$, that is $S_{\lambda, q}(y) \subset S_{\lambda, q}(x)$. 
Remark 7 Replacing the pseudo-quasimetric $q$ by the equivalent one $\lambda \cdot q$, we can suppose in all proofs that $\lambda=1$, that is we can work with the set-valued mapping $S$ given by

$$
S(x):=S_{1, q}(x)=\{y \in X \mid q(x, y) \leq \varphi(x)-\varphi(y)\} .
$$

Definition 7 (generalized-Picard sequences, Dancs et al. 1983, see also Bao and Théra 2015). Let $X$ be a nonempty set, $S: X \rightrightarrows X$ a set-valued mapping, and $\varphi: X \rightarrow \mathbb{R} \cup\{+\infty\}$ a function. We say that a sequence $\left\{x_{n}\right\}$ in $X$ is a GENERALIZED PICARD SEQUENCE ${ }^{12}$ of $S$, if $x_{n+1} \in S\left(x_{n}\right)$ for all $n \in \mathbb{N}$. It is said to be STRICTLY $\varphi$-DECREASING if the sequence $\left\{\varphi\left(x_{n}\right)\right\}$ is strictly decreasing, i.e., $\varphi\left(x_{n+1}\right)<\varphi\left(x_{n}\right)$ for all $n \in \mathbb{N}$.

Definition 8 (series completeness) A sequence $\left\{x_{n}\right\}$ in a pseudo-quasimetric space $(X, q)$ is said to be FORWARD- DISTANCE- SERIES- CONVERGENT if the forward-distance series $\sum_{n=1}^{\infty} q\left(x_{n}, x_{n+1}\right)$ is convergent. A pseudo-quasimetric space is said to be FORWARDDISTANCE- SERIES- COMPLETE if every forward-distance-series-convergent sequence is forward-convergent.

Proposition 3 Let $(X, q)$ be a pseudo-quasimetric space. The following hold:

(1) Every forward-distance-series-convergent sequence $\left\{x_{n}\right\}$ in $X$ is forward-Cauchy.

(2) The pseudo-quasimetric space $(X, q)$ is forward-forward-complete if and only if it is forward-distance-series-complete.

Proof (1) Consider an arbitrary forward-distance-series-convergent sequence $\left\{x_{n}\right\}$ in $X$, i.e., $\sum_{n=1}^{\infty} q\left(x_{n}, x_{n+1}\right)<\infty$. Then, for every $\varepsilon>0$, there is $N_{\varepsilon} \in \mathbb{N}$ such that for all $m \geq N_{\varepsilon}$ and for all $k \in \mathbb{N}$ we have

$$
q\left(x_{m}, x_{m+k}\right) \leq \sum_{n=m}^{m+k-1} q\left(x_{n}, x_{n+1}\right) \leq \sum_{n=m}^{\infty} q\left(x_{n}, x_{n+1}\right)<\varepsilon,
$$

i.e., $\left\{x_{n}\right\}$ is a forward-Cauchy sequence in $(X, q)$.

(2) Suppose that the space $(X, q)$ is forward-forward-complete. If the sequence $\left\{x_{n}\right\}$ satisfies the condition $\sum_{n=1}^{\infty} q\left(x_{n}, x_{n+1}\right)<\infty$, then, by (1), it is forward-Cauchy, so it is forwardconvergent to some $x_{*} \in X$.

Suppose now that the space $(X, q)$ is forward-distance-series-complete and let $\left\{x_{n}\right\}$ be forward-Cauchy. Let $n_{1} \in \mathbb{N}$ be such that

$$
q\left(x_{n}, x_{m}\right)<\frac{1}{2}, \forall m, n \in \mathbb{N}: n_{1} \leq n<m .
$$

Suppose that $n_{1}<n_{2}<\cdots<n_{k}$ are such that

$$
q\left(x_{n}, x_{m}\right)<\frac{1}{2^{i}}, \forall m, n \in \mathbb{N}: n_{i} \leq n<m, 1 \leq i \leq k .
$$

It follows $q\left(x_{n_{i}}, x_{n_{i+1}}\right)<\frac{1}{2^{i}}$ for $1 \leq i \leq k-1$. Let $n_{k+1}^{\prime}$ be such that

$$
q\left(x_{n}, x_{m}\right)<\frac{1}{2^{k+1}}, \forall m, n \in \mathbb{N}: n_{k+1}^{\prime} \leq n<m .
$$

\footnotetext{
12 The Picard-Lindelöf theorem, which shows that ordinary differential equations have solutions, is essentially an application of the Banach fixed point theorem to a special sequence of functions which forms a fixed point iteration, constructing the solution to the equation. Solving an ODE in this way is called Picard iteration, Picard's method, or the Picard iterative process.
} 
Taking $n_{k+1}:=1+\max \left\{n_{k}, n_{k+1}^{\prime}\right\}>n_{k}$, it follows $q\left(x_{n_{k}}, x_{n_{k+1}}\right)<\frac{1}{2^{k}}$. Also (3.4) holds with $n_{k+1}$ instead of $n_{k+1}^{\prime}$.

Continuing in this manner, we find the numbers $n_{1}<n_{2}<\ldots$ such that $q\left(x_{n_{k}}, x_{n_{k+1}}\right)<$ $1 / 2^{k}$ for all $k \in \mathbb{N}$, implying $\sum_{k=1}^{\infty} q\left(x_{n_{k}}, x_{n_{k+1}}\right)<1<\infty$. By hypothesis, the subsequence $\left\{x_{n_{k}}\right\}$ is forward- convergent to some $x_{*} \in X$. But then, by Theorem 1, the sequence $\left\{x_{n}\right\}$ is forward-convergent to $x_{*}$.

Remark 8 The construction from the last part of the proof is standard in metric spaces. In the case of quasimetric spaces, we have to take into account the asymmetry of the metric.

The following proposition contains some properties of generalized Picard sequences. We use the notation $\mathbb{N}=\{1,2, \ldots\}$ and $\mathbb{N}_{0}:=\mathbb{N} \cup\{0\}$.

Proposition 4 Let $(X, q)$ be a pseudo-quasimetric space, $\varphi: X \rightarrow \mathbb{R} \cup\{+\infty\}$ and $\left\{x_{n}\right\}_{n=0}^{\infty}$ a generalized Picard sequence of $S_{\lambda, q}$, with $x_{0} \in \operatorname{dom} \varphi$. Then, the following hold:

(1) The sequence $\left\{\varphi\left(x_{n}\right)\right\}_{n=0}^{\infty}$ is decreasing, i.e., $\varphi\left(x_{n+1}\right) \leq \varphi\left(x_{n}\right), \forall n \in \mathbb{N}_{0}$.

(2) If $\varphi$ is bounded from below on $S_{\lambda, q}\left(x_{0}\right)$, then the sequence $\left\{x_{n}\right\}$ is also forward-distanceseries-convergent.

Proof Put $S(x)=S_{1, q}(x)$. (1) The inequalities

$$
q\left(x_{k}, x_{k+1}\right) \leq \varphi\left(x_{k}\right)-\varphi\left(x_{k+1}\right) \quad\left(\Longleftrightarrow x_{k+1} \in S\left(x_{k}\right)\right)
$$

imply $\varphi\left(x_{k+1}\right) \leq \varphi\left(x_{k}\right), \forall k \in \mathbb{N}_{0}$. (2) By Proposition $2, S\left(x_{n+1}\right) \subset S\left(x_{n}\right) \subset S\left(x_{0}\right)$, so that $\inf \varphi\left(S\left(x_{0}\right)\right) \leq \inf \varphi\left(S\left(x_{n}\right)\right)$ for all $n \in \mathbb{N}$, and the above inequalities yield by summation

$$
\sum_{k=0}^{n} q\left(x_{k}, x_{k+1}\right) \leq \varphi\left(x_{0}\right)-\varphi\left(x_{n+1}\right) \leq \varphi\left(x_{0}\right)-\inf \varphi\left(S\left(x_{n+1}\right)\right) \leq \varphi\left(x_{0}\right)-\inf \varphi\left(S\left(x_{0}\right)\right),
$$

showing that the sequence $\left\{x_{n}\right\}_{n \in \mathbb{N}_{0}}$ is forward-distance-series-convergent, provided $\varphi$ is bounded below on $S\left(x_{0}\right)$.

We can state now the first version of Ekeland's variational principle.

Theorem 4 (a forward version of Ekeland's variational principle) Let $(X, q)$ be a pseudoquasimetric space and $\varphi: X \rightarrow \mathbb{R} \cup\{+\infty\}$ be a function. Given $\lambda>0$. Suppose that there exists $x_{0} \in \operatorname{dom}(\varphi)$ such that

(C1) $\inf \varphi\left(S_{\lambda, q}\left(x_{0}\right)\right)>-\infty$ (i.e. $\varphi$ is bounded from below on $S_{\lambda, q}\left(x_{0}\right)$ );

(C2) for every sequence $\left\{x_{n}\right\}_{n=0}^{\infty}$ which is forward-distance-series-convergent, generalized Picard with respect to $S_{\lambda, q}$ (starting with $x_{0}$ ), and strictly $\varphi$-decreasing, there exists $y \in X$ such that $S_{\lambda, q}(y) \subset \bigcap_{n=0}^{\infty} S_{\lambda, q}\left(x_{n}\right)$.

Then there exists a forward-distance-series-convergent sequence $\left\{x_{n}\right\}_{n \in \mathbb{N}_{0}}$ which is generalized Picard with respect to $S_{\lambda, q}$ and forward-convergent to some $x_{*} \in X$ such that for every $y_{*} \in S_{\lambda, q}\left(x_{*}\right)$ the following conditions hold:

$$
\begin{aligned}
\text { (i) } & \varphi\left(y_{*}\right)+\lambda q\left(x_{0}, y_{*}\right) \leq \varphi\left(x_{0}\right) ; \\
\text { (ii) } & \varphi(x)+\lambda q\left(y_{*}, x\right)>\varphi\left(y_{*}\right), \forall x \in X \backslash S_{\lambda, q}\left(y_{*}\right) ; \\
\text { (iii) } & q\left(x_{*}, y_{*}\right)=0, S_{\lambda, q}\left(y_{*}\right) \subset \overline{\left\{y_{*}\right\}}, \\
& \varphi(x)=\varphi\left(y_{*}\right)=\varphi\left(x_{*}\right), \forall x \in S_{\lambda, q}\left(y_{*}\right), \text { and } \\
& \varphi(x)>\varphi\left(y_{*}\right), \forall x \in \overline{\left\{y_{*}\right\}} \backslash S_{\lambda, q}\left(y_{*}\right) .
\end{aligned}
$$


Proof Replacing, if necessary, $q$ by $\lambda q$, we can suppose $\lambda=1$. Put also $S(x)=S_{1, q}(x), x \in$ $X$.

Case I. We shall define inductively a forward-distance-series-convergent generalized Picard sequence which will satisfy all the requirements of the theorem.

Start with $x_{0}$ and suppose that $\alpha_{0}:=\inf \varphi\left(S\left(x_{0}\right)\right)<\varphi\left(x_{0}\right)$. Choose $x_{1} \in S\left(x_{0}\right)$ such that

$$
\alpha_{0} \leq \varphi\left(x_{1}\right)<\alpha_{0}+\frac{1}{2}\left(\varphi\left(x_{0}\right)-\alpha_{0}\right)=\frac{1}{2}\left(\alpha_{0}+\varphi\left(x_{0}\right)\right)<\varphi\left(x_{0}\right) .
$$

Suppose that we have found $x_{0}, x_{1}, \ldots, x_{n}$ satisfying

$$
\begin{aligned}
& x_{k+1} \in S\left(x_{k}\right), \\
& \alpha_{k} \leq \varphi\left(x_{k+1}\right)<\alpha_{k}+\frac{1}{2}\left(\varphi\left(x_{k}\right)-\alpha_{k}\right)=\frac{1}{2}\left(\alpha_{k}+\varphi\left(x_{k}\right)\right)<\varphi\left(x_{k}\right),
\end{aligned}
$$

for $k=0,1, \ldots, n-1$, and $\varphi\left(x_{k}\right)>\alpha_{k}:=\inf \varphi\left(S\left(x_{k}\right)\right), k=0,1, \ldots, n$.

Pick then $x_{n+1} \in S\left(x_{n}\right)$ such that

$$
\alpha_{n} \leq \varphi\left(x_{n+1}\right)<\alpha_{n}+\frac{1}{2}\left(\varphi\left(x_{n}\right)-\alpha_{n}\right)=\frac{1}{2}\left(\alpha_{n}+\varphi\left(x_{n}\right)\right)<\varphi\left(x_{n}\right) .
$$

Supposing that we can do indefinitely this procedure, we find a generalized Picard sequence $x_{n+1} \in S\left(x_{n}\right)$ satisfying (3.6) for all $n \in \mathbb{N}_{0}$. Let us show that the conditions from (3.5) are satisfied by this sequence.

Since the sequence $\left\{\varphi\left(x_{n}\right)\right\}$ is strictly decreasing and bounded from below (by condition (C1)), there exists $\alpha_{*}:=\lim _{n \rightarrow \infty} \varphi\left(x_{n}\right)=\inf _{n \rightarrow \infty} \varphi\left(x_{n}\right)$.

By Proposition 2, $x_{n+1} \in S\left(x_{n}\right)$ implies $S\left(x_{n+1}\right) \subset S\left(x_{n}\right) \subset S\left(x_{0}\right)$, so that $\alpha_{n+1} \geq \alpha_{n}$, implying the existence of $\alpha=\lim _{n \rightarrow \infty} \alpha_{n}$. The inequalities

$$
\varphi\left(x_{n+1}\right)<\frac{1}{2}\left(\alpha_{n}+\varphi\left(x_{n}\right)\right)<\varphi\left(x_{n}\right)
$$

yield for $n \rightarrow \infty, \alpha_{*} \leq \frac{1}{2}\left(\alpha+\alpha_{*}\right) \leq \alpha_{*}$, implying $\alpha=\alpha_{*}$. Consequently

$$
\lim _{n \rightarrow \infty} \alpha_{n}=\lim _{n \rightarrow \infty} \varphi\left(x_{n}\right)=\alpha_{*} .
$$

Since $\varphi$ is bounded below on $S\left(x_{0}\right)$, Proposition 4 implies that the generalized Picard sequence $\left\{x_{n}\right\}$ is forward-distance-series-convergent. Since the sequence $\left\{\varphi\left(x_{n}\right)\right\}$ is also strictly decreasing, condition (C2) implies the existence of a point $x_{*} \in X$ such that

$$
S\left(x_{*}\right) \subset \bigcap_{n=0}^{\infty} S\left(x_{n}\right) .
$$

Since $x_{*} \in S\left(x_{*}\right) \subset S\left(x_{n}\right)$, it follows that, for all $n \in \mathbb{N}_{0}$,

$$
0 \leq q\left(x_{n}, x_{*}\right) \leq \varphi\left(x_{n}\right)-\varphi\left(x_{*}\right) .
$$

Consequently,

$$
\alpha_{n} \leq \varphi\left(x_{*}\right) \leq \varphi\left(x_{n}\right), \forall n \in \mathbb{N}_{0},
$$

yielding for $n \rightarrow \infty, \varphi\left(x_{*}\right)=\alpha_{*}$. But then, the inequalities (3.7) imply $\lim _{n \rightarrow \infty} q\left(x_{n}, x_{*}\right)=$ 0 , that is the sequence $\left\{x_{n}\right\}$ is forward-convergent to $x_{*}$.

Let $y_{*} \in S\left(x_{*}\right)$. Condition (i) is equivalent to $y_{*} \in S\left(x_{0}\right)$, which is true because $y_{*} \in$ $S\left(x_{*}\right) \subset S\left(x_{0}\right)$. Condition (ii) follows from the definition of the set $S\left(y_{*}\right)$. Let us show that the conditions from (iii) are also satisfied. 
The relations $y_{*} \in S\left(x_{*}\right) \subset S\left(x_{n}\right)$ imply $\alpha_{n} \leq \varphi\left(y_{*}\right) \leq \varphi\left(x_{*}\right)=\alpha_{*}, \forall n \in \mathbb{N}_{0}$. Letting $n \rightarrow \infty$, one obtains $\varphi\left(y_{*}\right)=\alpha_{*}=\varphi\left(x_{*}\right)$. Also

$$
y_{*} \in S\left(x_{*}\right) \Longleftrightarrow 0 \leq q\left(x_{*}, y_{*}\right) \leq \varphi\left(x_{*}\right)-\varphi\left(y_{*}\right)=0,
$$

so that $q\left(x_{*}, y_{*}\right)=0$.

If $x \in S\left(y_{*}\right) \subset S\left(x_{*}\right)$, then, as above, $\alpha_{n} \leq \varphi(x) \leq \varphi\left(x_{*}\right)=\alpha_{*}, \forall n \in \mathbb{N}_{0}$, yields for $n \rightarrow \infty, \varphi(x)=\varphi\left(x_{*}\right)=\varphi\left(y_{*}\right)$. The inequality $0 \leq q\left(y_{*}, x\right) \leq \varphi\left(y_{*}\right)-\varphi(x)=0$ implies $q\left(y_{*}, x\right)=0$, that is $x \in{\overline{\left\{y_{*}\right\}}}^{\bar{q}}$.

If $x \in \overline{\left\{y_{*}\right\}} \bar{q} \backslash S_{\lambda, q}\left(y_{*}\right)$, then $q\left(y_{*}, x\right)=0$ and, by (ii), $\varphi\left(y_{*}\right)<\varphi(x)+\lambda q\left(y_{*}, x\right)=\varphi(x)$.

Case II. Suppose that, for some $n_{0} \in \mathbb{N}_{0}, \varphi\left(x_{n_{0}}\right)=\alpha_{n_{0}}=\inf \varphi\left(S\left(x_{n_{0}}\right)\right)$. Then take $x_{n_{0}+1}=$ $x_{n_{0}}$ and, by induction, $x_{n_{0}+k}=x_{n_{0}}$ for all $k \in \mathbb{N}$.

Then the sequence $\left\{x_{n}\right\}$ is forward-distance-series-convergent with

$$
\sum_{k=0}^{\infty} q\left(x_{k}, x_{k+1}\right)=\sum_{k=0}^{n_{0}-1} q\left(x_{k}, x_{k+1}\right),
$$

and forward-convergent to $x_{n_{0}}$. Also, for $x \in S\left(x_{n_{0}}\right), \varphi(x) \geq \alpha_{n_{0}}=\varphi\left(x_{n_{0}}\right)$, so that the inequalities

$$
0 \leq q\left(x_{n_{0}}, x\right) \leq \varphi\left(x_{n_{0}}\right)-\varphi(x) \leq 0,
$$

imply $q\left(x_{n_{0}}, x\right)=0$ and $\varphi\left(x_{n_{0}}\right)=\varphi(x)$. It follows also that $x \in{\overline{\left\{x_{n_{0}}\right.}}^{\bar{q}}$, that is $S\left(x_{n_{0}}\right) \subset$ $\overline{\left\{x_{n_{0}}\right\}^{q}}$.

These show that condition (iii) is satisfied. The validity of conditions (i) and (ii) follows as in Case I.

Remark 9 Conditions (ii) and (iii) from Theorem 4 imply

(ii') $\quad \varphi\left(y_{*}\right)<\varphi(x)+\lambda \rho\left(y_{*}, x\right), \forall x \in X \backslash{\overline{\left\{y_{*}\right.}}^{\bar{q}}$.

(iii') $\varphi\left(y_{*}\right) \leq \varphi(x), \forall x \in{\overline{\left\{y_{*}\right\}}}^{\bar{q}}$.

Similar conditions appear in Karapinar and Romaguera (2015, Theorem 2).

Corollary 1 (a simple version in pseudo-quasimetric spaces) Assume that all the hypotheses in Theorem 4 hold. Assume in addition that

(C3) (forward-limit uniqueness) any generalized-Picard sequence $\left\{x_{n}\right\}$ of $S_{\lambda, q}$, being strictly $\varphi$-decreasing and forward-distance-series-convergent, has at most one forward-limit.

Then, there is $x_{*} \in S_{\lambda, q}\left(x_{0}\right)$ such that $S_{\lambda, q}\left(x_{*}\right)=\left\{x_{*}\right\}$, conditions which are respectively equivalent to

(i) $\varphi\left(x_{*}\right)+\lambda q\left(x_{0}, x_{*}\right) \leq \varphi\left(x_{0}\right)$;

(ii) $\varphi(x)+\lambda q\left(x_{*}, x\right)>\varphi\left(x_{*}\right), \quad \forall x \neq x_{*}$.

Proof It is straightforward from Theorem 4.

Employing Theorem 4 in the context of the pseudo-quasimetric space $(X, \bar{q})$, where $\bar{q}(x, y)=q(y, x), x, y \in X$, we obtain also a backward version of Ekeland's variational principle. 
Corollary 2 (a backward version of Ekeland's variational principle) Let $(X, q)$ be a pseudoquasimetric space, and $\varphi: X \rightarrow \mathbb{R} \cup\{+\infty\}$ a proper extended-real-valued function. Given $x_{0} \in \operatorname{dom} \varphi$ and $\lambda>0$, consider the set-valued mapping $S_{\lambda, \bar{q}}: X \rightrightarrows X$ defined by

$$
S_{\lambda, \bar{q}}(x):=\{y \in X \mid \varphi(y)+\lambda q(y, x) \leq \varphi(x)\}, \forall x \in X .
$$

Impose the boundedness from below condition (C1) from Theorem 4 and the backward nonempty intersection condition

(C2B) For any generalized-Picard sequence $\left\{x_{n}\right\}_{n=0}^{\infty}$ of $S_{\lambda, \bar{q}}$, being strictly $\varphi$-decreasing and backward-distance-series-convergent (i.e. $\left.\sum_{n=0}^{\infty} q\left(x_{n+1}, x_{n}\right)<\infty\right)$, there exists $y \in X$ such that $S_{\lambda, \bar{q}}(y) \subset S_{\lambda, \bar{q}}\left(x_{n}\right)$ for all $n \in \mathbb{N}_{0}$.

Then, there is $x_{*} \in S_{\lambda, \bar{q}}\left(x_{0}\right)$ such that for every $y_{*} \in S_{\lambda, \bar{q}}\left(x_{*}\right)$ one has

$$
\begin{aligned}
\text { (i) } & \varphi\left(y_{*}\right)+\lambda q\left(y_{*}, x_{0}\right) \leq \varphi\left(x_{0}\right) ; \\
\text { (ii) } & \varphi(x)+\lambda q\left(x, y_{*}\right)>\varphi\left(y_{*}\right), \quad \forall x \in X \backslash{\overline{\left\{y_{*}\right\}}}^{q}: \\
\text { (iii) } & q\left(x_{*}, y_{*}\right)=0, S_{\lambda, \bar{q}}\left(y_{*}\right) \subset \overline{\left\{y_{*}\right\}}, \\
& \varphi(x)=\varphi\left(y_{*}\right)=\varphi\left(x_{*}\right), \forall x \in S_{\lambda, \bar{q}}\left(y_{*}\right), \text { and } \\
& \varphi(x)>\varphi\left(y_{*}\right), \forall x \in{\overline{\left\{y_{*}\right\}}}^{q} \backslash S_{\lambda, \bar{q}}\left(y_{*}\right) .
\end{aligned}
$$

where ${\overline{\left\{y_{*}\right\}}}^{q}=\left\{u \in X \mid q\left(u, y_{*}\right)=0\right\}$.

Proof It follows from Theorem 4 by using the pseudo-quasimetric space $(X, \bar{q})$ instead of $(X, q)$.

Let us revisit Examples 3-5.

\section{Example 6 (Examples 3-5 (revisited)).}

- Since Condition (C2) in Example 3 does not hold, both Theorem 4 and its Corollary 1 are not applicable. Precisely, we have $\varphi(x)=e^{x}, S_{1, q_{1}}(x)=(-\infty, x],\left\{x_{n}\right\}$ with $x_{n}=-n$ is a strict- $\varphi$-decreasingly forward-distance-series-convergent generalized-Picard sequence of $S_{1, q_{1}}$, but for any number $y \in \mathbb{R}$ one has

$$
S_{1, q_{1}}(y)=(-\infty, y] \not \subset S_{1, q_{1}}\left(x_{n}\right)=(-\infty,-n] \text { whenever }-n<y
$$

clearly verifying that the nonempty intersection condition (C2) is not fulfilled.

- In Example 4, it is easy to check the validity of the bounded condition (C1) and the nonempty intersection condition (C2) with $y=-1$ for any strict- $\varphi$-decreasingly forwarddistance-series-convergent generalized-Picard sequence of $S_{\lambda, q_{1}}$.

Take $\lambda=2$ and $x_{0}=1$. Then, the sequence $x_{n} \equiv x_{*}$ for all $n \in \mathbb{N}$, where $x_{*}$ and 1 are two distinct solutions of the equation $e^{x}=2(x-1)+e$, satisfies both the assertions (i) and (ii) in Theorem 4 which, respectively, reduce to

(i) $e^{x_{*}}+2 q_{1}\left(1, x_{*}\right)=2^{1}$;

(ii) $\varphi(x)+2 q_{1}\left(x_{*}, x\right)>\varphi\left(x_{*}\right), \quad \forall x \in[-1,1] \backslash \overrightarrow{\left\{x_{n}\right\}}=\left(x_{*}, 1\right]$.

Note that excluding all the forward-limits in (ii) is essential since for any $x<x_{*}$, one has $\varphi(x)+2 q_{1}\left(x_{*}, x\right)=\varphi(x)<\varphi\left(x_{*}\right)$, i.e., (ii) is not valid.

Take $\lambda=1$ and $x_{0}=0$. Then, the desired point of Theorem 4 is then $x_{*}=0$. 
- In Example 5, it is easy to check the validity of condition (C2). For any strict- $\varphi$ decreasingly forward-distance-series-convergent generalized-Picard sequence $\left\{x_{n}\right\}$ in $\mathbb{R}$, the sequence $\left\{x_{n}\right\}$ is a Cauchy sequence in the complete metric space $(\mathbb{R},|\cdot|)$. Therefore, the classical Ekeland's variational principle applied to the closed set $\left\{x_{n}\right\} \cup\left\{x_{*}\right\}$ ensures the existence of some element $x_{*}$ satisfying condition (C2).

Next, we will provide an efficient sufficient condition for the nonempty intersection condition (C2) which is less restrictive than lower semicontinuity.

Definition 9 (strict-decreasingly forward-lsc functions) Let $(X, q)$ be a pseudo-quasimetric space and $\varphi: X \rightarrow \mathbb{R} \cup\{+\infty\}$ be an extended real-valued function on $X$.

(i) The function $\varphi$ is said to be STRICT- DECREASINGLY FORWARD- LOWER- SEMICONTINUOUS, if for every forward-convergent sequence $\left\{x_{n}\right\}$ such that $\left\{\varphi\left(x_{n}\right)\right\}$ is strictly decreasing, one has

$$
\varphi\left(y_{*}\right) \leq \lim _{n \rightarrow \infty} \varphi\left(x_{n}\right), \forall y_{*} \in \overrightarrow{\left\{x_{n}\right\}},
$$

where $\overrightarrow{\left\{x_{n}\right\}}=\left\{y_{*} \in X \mid \lim _{n \rightarrow \infty} q\left(x_{n}, y_{*}\right)=0\right\}$ is the collection of forward-limits of the sequence $\left\{x_{n}\right\}$.

(ii) A related notion is that of DECREASINGLY FORWARD- LSC FUNCTION considered by Kirk and Saliga (2001) (called by them lower-semicontinuity from above) meaning that $\varphi\left(x_{*}\right) \leq \lim _{n \rightarrow \infty} \varphi\left(x_{n}\right)$ for every sequence $\left\{x_{n}\right\}$ forward-convergent to $x_{*}$ and such that $\varphi\left(x_{n+1}\right) \leq \varphi\left(x_{n}\right), \forall n \in \mathbb{N}$.

(iii) Following Karapinar and Romaguera (2015) we call the function $\varphi$ FORWARDNEARLY- LSC if, whenever a sequence $\left\{x_{n}\right\}$ of distinct points in $X$ is forward-convergent to a forward-limit $x_{*}$, then $\varphi\left(x_{*}\right) \leq \liminf _{n \rightarrow \infty} \varphi\left(x_{n}\right)$.

Similarly, we could define the concepts of strict-decreasing backward-lower-semicontinuity, of decreasing backward-lower-semicontinuity, and of backward-near-lower-semicontinuity.

It is worth mentioning that the class of strict-decreasingly forward-lower-semicontinuous functions is broader than the union of that of the decreasingly forward-lsc functions and that of the nearly lsc functions. Since the strict- $\varphi$-decreasing requirement of the sequence $\left\{x_{n}\right\}$ implies that $x_{n} \neq x_{m}$ for all $n \neq m$, every nearly lsc function is strict- $\varphi$-decreasingly forward-lsc.

Let us provide some examples with functions satisfying these conditions.

\section{Example 7 (decreasingly forward-lsc, strict-decreasingly forward-lsc and lsc functions)}

- Consider the functions $\varphi, \varphi_{1}:(\mathbb{R},|\cdot|) \rightarrow(\mathbb{R},|\cdot|)$ given by

$$
\varphi(x):=\left\{\begin{array}{ll}
x & \text { if } x \geq 0 \\
-1 & \text { if } x<0
\end{array} \text { and } \varphi_{1}(x):= \begin{cases}-x & \text { if } x>0 \\
1 & \text { if } x \leq 0 .\end{cases}\right.
$$

Then $\varphi$ is strict-decreasingly forward ${ }^{13}$-lsc at 0 , but not decreasingly lsc (and so not lsc) at 0 , since the sequence $x_{n}=-1 / n, n \in \mathbb{N}$, is convergent to 0 and $\varphi\left(x_{n}\right)=-1$ for all $n \in \mathbb{N}$, so that $\lim _{n \rightarrow \infty} \varphi\left(x_{n}\right)=-1<0=\varphi(0)$. The function $\varphi_{1}$ is decreasingly forward-lsc at 0 , but not lsc. Indeed, there are no sequences $x_{n} \rightarrow 0$ with $\left\{\varphi_{1}\left(x_{n}\right)\right\}$ strictly decreasing. If $x_{n} \rightarrow 0$ and $\varphi_{1}\left(x_{n+1}\right) \leq \varphi_{1}\left(x_{n}\right)$ for all $n$, then $\varphi_{1}\left(x_{n}\right)=1$ for sufficiently large $n$, so that $\lim _{n \rightarrow \infty} \varphi_{1}\left(x_{n}\right)=1=\varphi(0)$. The function $\varphi_{1}$ is not lsc at 0 because $\lim _{x \searrow 0} \varphi_{1}(x)=0<1=\varphi_{1}(0)$.

13 The space in question is a metric space and thus there is no difference between two topologies. 
- The function $\varphi(x)=x$ is not strict-decreasingly forward-lsc in the quasimetric space $\left([0,1], q_{4}\right)^{14}$, where $q_{4}$ is defined by

$$
q_{4}(x, y)= \begin{cases}x-y & \text { if } x \geq y \\ 1+x-y & \text { if } x<y \text { but }(x, y) \neq(0,1) \\ 1 & \text { if }(x, y)=(0,1)\end{cases}
$$

In this space, the strict- $\varphi$-decreasingly forward-Cauchy sequence $\left\{x_{n}\right\}$ with $x_{n}=\frac{1}{n}$ has two forward-limits $x_{*}=0$ and $y_{*}=1$. Obviously,

$$
\liminf _{n \rightarrow \infty} \varphi\left(x_{n}\right)=\lim _{n \rightarrow \infty} \frac{1}{n}=0=\varphi(0)=\varphi\left(x_{*}\right) \neq \varphi(1)=\varphi\left(y_{*}\right) .
$$

This proves that $\varphi$ is not strict-decreasingly lsc in $\left(X, q_{4}\right)$. Incidentally, this furnishes another example of a quasimetric space (hence $T_{1}$ ) where the uniqueness condition for forward-limits does not hold (see Example 2). It is easy to check that the sequence $x_{n}=1 / n$ is also forward-Cauchy.

- The everywhere discontinuous function $\varphi(x)=0$ for $x \in \mathbb{Q}$ and $\varphi(x)=1$ for $x \in \mathbb{R} \backslash \mathbb{Q}$, defined on $(\mathbb{R},|\cdot|)$, is strict-decreasingly lsc because there are no strictly $\varphi$-decreasing sequences. The function $\varphi$ is not lsc at every point $x \in \mathbb{R} \backslash \mathbb{Q}$, because $\varphi(x)=1>0=$ $\liminf _{u \rightarrow x} \varphi(u)$. Also, it is not upper-semicontinuous at every $x \in \mathbb{Q}$.

We shall present now some completeness conditions guaranteeing the validity of condition (C2) from Theorem 4. Consider also the condition

$\left(\mathrm{C} 2^{\prime}\right)$ for every sequence $\left\{x_{n}\right\}_{n=0}^{\infty}$, generalized Picard with respect to $S_{\lambda, q}$, there exists $y \in X$ such that $S_{\lambda, q}(y) \subset \bigcap_{n=0}^{\infty} S_{\lambda, q}\left(x_{n}\right)$,

where $S_{\lambda, q}(x)$ is defined by (3.2).

By Proposition $3,\left(\mathrm{C}^{\prime}\right) \Rightarrow(\mathrm{C} 2)$.

Lemma 1 Let $(X, q)$ be a pseudo-quasimetric space, $\varphi: X \rightarrow \mathbb{R} \cup\{+\infty\}$ a proper function, $x_{0} \in \operatorname{dom} \varphi$, and $\left\{x_{n}\right\}_{n=0}^{\infty}$ a generalized Picard sequence of $S_{\lambda, q}$ forward-convergent to some $x_{*}$.

(1) If the function $\varphi$ is decreasingly forward-lsc on $\operatorname{dom} \varphi$ then

$$
x_{*} \in \bigcap_{n=0}^{\infty} S_{\lambda, q}\left(x_{n}\right) .
$$

By Proposition 2 this implies $S_{\lambda, q}\left(x_{*}\right) \subset \bigcap_{n=0}^{\infty} S_{\lambda, q}\left(x_{n}\right)$.

(2) If the function $\varphi$ is strict-decreasingly forward-lsc on $\operatorname{dom} \varphi$, then (3.8) holds if, in addition, the sequence $\left\{x_{n}\right\}$ is strictly $\varphi$-decreasing.

Proof Work again with the sets $S(x)=S_{1, q}(x)$ (as in the proof of Theorem 4).

Since

$$
x_{k+1} \in S\left(x_{k}\right) \Longleftrightarrow q\left(x_{k}, x_{k+1}\right) \leq \varphi\left(x_{k}\right)-\varphi\left(x_{k+1}\right),
$$

it follows that the sequence $\left\{\varphi\left(x_{n}\right)\right\}_{n=0}^{\infty}$ is decreasing and

$$
q\left(x_{n}, x_{n+k}\right) \leq \varphi\left(x_{n}\right)-\varphi\left(x_{n+k}\right)
$$

14 The conjugate quasimetric of the one studied in Lin et al. (2011, Example 3.16). 
so that

$$
q\left(x_{n}, x_{*}\right) \leq q\left(x_{n}, x_{n+k}\right)+q\left(x_{n+k}, x_{*}\right) \leq \varphi\left(x_{n}\right)-\varphi\left(x_{n+k}\right)+q\left(x_{n+k}, x_{*}\right), \forall n, k \in \mathbb{N}_{0} .
$$

Letting $k \rightarrow \infty$ and taking into account that $\varphi\left(x_{*}\right) \leq \lim _{k \rightarrow \infty} \varphi\left(x_{n+k}\right)$ and $\lim _{k \rightarrow \infty} q\left(x_{n+k}, x_{*}\right)=0$, one obtains

$$
q\left(x_{n}, x_{*}\right) \leq \varphi\left(x_{n}\right)-\varphi\left(x_{*}\right) \Longleftrightarrow x_{*} \in S\left(x_{n}\right), \forall n \in \mathbb{N}_{0} .
$$

The same proof works in the case that the function $\varphi$ is strict-decreasingly forward-lsc.

Proposition 5 (sufficient conditions for $(\mathrm{C} 2)$ and $\left.\left(\mathrm{C}^{\prime}\right)\right)$ Let $(X, q)$ be a pseudo-quasimetric space and $\varphi: X \rightarrow \mathbb{R} \cup\{+\infty\}$ a proper function.

(1) If every forward-distance-series-convergent and strictly $\varphi$-decreasing sequence in the space $(X, q)$ is forward-convergent and the function $\varphi$ is strict-decreasingly forward-lsc on $\operatorname{dom} \varphi$, then condition $(\mathrm{C} 2)$ is satisfied.

(2) If the space $(X, q)$ is forward-distance-series-complete and the function $\varphi$ is decreasingly forward-lsc on $\operatorname{dom} \varphi$ and bounded from below, then condition $\left(\mathrm{C}^{\prime}\right)$ is satisfied.

Proof Let $x_{0} \in \operatorname{dom} \varphi$ and $\left\{x_{n}\right\}_{n=0}^{\infty}$ a generalized Picard sequence with respect to $S_{\lambda, q}$.

(1) If the sequence $\left\{x_{n}\right\}$ is forward-distance-series-convergent and strictly $\varphi$-decreasing, then, by the completeness hypothesis, it has a forward-limit $x_{*}$. By Lemma 1 it follows $S_{\lambda, q}\left(x_{*}\right) \subset \bigcap_{n=0}^{\infty} S_{\lambda, q}\left(x_{n}\right)$, i.e. condition (C2) is satisfied.

(2) The generalized Picard sequence $\left\{x_{n}\right\}$ is $\varphi$-decreasing, so that, by Proposition 4 , it is forward-distance-series-convergent. By hypothesis it has a forward-limit $x_{*}$. We can apply again Lemma 1 to conclude that $S_{\lambda, q}\left(x_{*}\right) \subset \bigcap_{n=0}^{\infty} S_{\lambda, q}\left(x_{n}\right)$, i.e. condition (C2') is satisfied.

Corollary 3 Let $(X, q)$ be a pseudo-quasimetric space and $\varphi: X \rightarrow \mathbb{R} \cup\{+\infty\}$ a proper extended-real-valued function. Assume that every forward-distance-series-convergent and strictly $\varphi$-decreasing sequence in the space $(X, q)$ is forward-convergent, the function $\varphi$ is proper, bounded from below, and strict-decreasingly forward-lsc. Then, for any $x_{0} \in \operatorname{dom} \varphi$ and $\lambda>0$, there is $x_{*} \in S_{\lambda, q}\left(x_{0}\right)$ such that for every $y_{*} \in S_{\lambda, q}\left(x_{*}\right)$, one has

$$
\begin{aligned}
\text { (i) } & \varphi\left(y_{*}\right)+\lambda q\left(x_{0}, y_{*}\right) \leq \varphi\left(x_{0}\right) ; \\
\text { (ii) } & \varphi(x)+\lambda q\left(y_{*}, x\right)>\varphi\left(y_{*}\right), \forall x \in X \backslash \overline{\left\{y_{*}\right\}} \bar{q} ; \\
\text { (iii) } & q\left(x_{*}, y_{*}\right)=0, S_{\lambda, q}\left(y_{*}\right) \subset \overline{\left\{y_{*}\right\}}, \\
& \varphi(x)=\varphi\left(y_{*}\right)=\varphi\left(x_{*}\right), \forall x \in S_{\lambda, q}\left(y_{*}\right), \text { and } \\
& \varphi(x)>\varphi\left(y_{*}\right), \forall x \in{\overline{\left\{y_{*}\right\}}}^{\bar{q}} \backslash S_{\lambda, q}\left(y_{*}\right) .
\end{aligned}
$$

Assume, in addition, that the uniqueness condition (C3) from Corollary 1 holds. Then, for any $x_{0} \in \operatorname{dom} \varphi$ and $\lambda>0$, there is $x_{*} \in X$ that satisfies conditions ( $i$ ) and (ii) of the same corollary.

Proof By Proposition 5, condition (C2) is satisfied. As the function $\varphi$ is bounded from below, we can apply Theorem 4 to conclude. 
Remark 10 The forward-limit uniqueness of strictly $\varphi$-decreasing sequences allows us to use the pseudo-quasimetric versions of Ekeland's variational principle when the space is not forward-complete; for example, the space $\left(\mathbb{R}, q_{1}\right)$, where $q_{1}(x, y)=y-x$ if $x \leq y$ and $q_{1}(x, y)=0$ otherwise (see Example 1). Consider the sequence $x_{n}=-n$ for all $n \in \mathbb{N}$. Then $q_{1}\left(x_{n}, x_{n+k}\right)=0$ for all $n, k \in \mathbb{N}$, so that $\left\{x_{n}\right\}$ is forward-Cauchy. However, this sequence has no forward-limit. Given $x^{*} \in \mathbb{R}, q_{1}\left(x_{n}, x_{*}\right)=x_{*}+n$ for all $n>x_{*}$ and thus $\lim _{n \rightarrow \infty} q_{1}\left(x_{n}, x_{*}\right)=+\infty$. This proves that $x^{*}$ is not a forward-limit of $\left\{x_{n}\right\}$. Since $x^{*}$ was arbitrary, the sequence has no forward-limit.

Remark 11 (a) Corollary 3 is more general than both Karapinar and Romaguera (2015, Theorem 2(3)) and Bao et al. (2015b, Corollary 3.3).

(b) Proceeding in a similar way, the backward nonempty intersection condition (C2B) is fulfilled provided that the cost function is strict-decreasingly backward-lsc. Thus, we could formulate a backward version of Corollary 3.

(c) The nonempty intersection condition (C2) is similar to the one used in Bao et al. (2015, Theorem 4.1). Assume that $(X, q)$ is a complete metric space, condition $(\mathrm{C} 2)$ reduces to

$\left(\mathrm{C}^{\prime \prime}\right)$ for any strict- $\varphi$-decreasingly forward-Cauchy generalized-Picard sequence $\left\{x_{n}\right\}$, one has $S_{\lambda, q}\left(y_{*}\right) \subset S_{\lambda, q}\left(x_{n}\right)$ for all $n \in \mathbb{N}$, where $y_{*}$ is the unique limit of the sequence $\left\{x_{n}\right\}$

since there is no difference between forward-Cauchy and backward-Cauchy sequences and the limit (if it exists) is unique. Condition (C2") was first used in Khanh and Quy (2013).

(d) Theorem 4 and its Corollaries 1 and 3 can be seen as far-going extended versions of Ekeland's variational principle in pseudo-quasi-metric spaces which might not be forward Hausdorff. In several less general settings, versions of Ekeland's variational principle were established by Bao (2015), Bao et al. (2015a,b,c, 2016) (forward Hausdorff ${ }^{15}$ quasimetric spaces), by Cobzaş (2011) ( $T_{1}$ quasimetric space ${ }^{16}$ is $T_{1}$ - for any distinct points $x$ and $y$ in $X$ each of them has a neighborhood not containing the other), by Ume (2002) (regular ${ }^{17}$ quasimetric spaces).

(e) Based on the fact that if $(X, p)$ is a partial pseudo-metric space, then the space $(X, q)$ with $q(x, y)=p(x, y)-p(y, y)$ is a pseudo-quasimetric space, it is possible to formulate new versions of Ekeland's variational principle as well as their equivalent fixed point theorems in partial pseudo-metric spaces. They are more general than the existing results in partial metric spaces; see Aydi et al. (2015).

It is well known that the original Ekeland's variational principle has been shown by Sullivan to be equivalent to the completeness of metric spaces. For an analysis of connections between various types of completeness, variational principles and fixed point theorems see the survey paper (Cobzaş 2016).

In the rest of this section, we will show that Corollary 3 yields a characterization of forward completeness of pseudo-quasimetric spaces.

To the best of our knowledge, Karapinar and Romaguera were the firsts who established a version of Ekeland's variational principle in Karapinar and Romaguera (2015) for

\footnotetext{
15 Known as 'left Hausdorff' in the cited papers.

16 The topology $\tau(\bar{q})$.

17 Cobzaş (2011, Remark 1.4) pointed out that the topology $\tau(q)$ is regular under the assumptions made in the cited paper.
} 
non-necessarily $T_{1}$ quasimetric $^{18}$ spaces, and proved that their weak version of Ekeland's variational principle is equivalent to the completeness of the corresponding quasimetric space.

Theorem 5 (Karapinar and Romaguera 2015, Theorem 2) For a pseudo-quasimetric space $(X, q)$ the following conditions are equivalent.

(1) $(X, q)$ is forward-forward complete.

(2) For every proper bounded below and forward-nearly-lsc function $f: X \rightarrow \mathbb{R} \cup\{+\infty\}$ and for every $\varepsilon>0$ there exists $y_{\varepsilon} \in X$ such that

$$
\begin{aligned}
& \text { (i) } f\left(y_{\varepsilon}\right) \leq \inf f(X)+\varepsilon \text {; } \\
& \text { (ii) } f\left(y_{\varepsilon}\right)<f(x)+\varepsilon q\left(y_{\varepsilon}, x\right), \forall x \in X \backslash \overline{\left\{y_{\varepsilon}\right\}} \bar{q} \text {, and } \\
& \text { (iii) } f\left(y_{\varepsilon}\right) \leq f(x), \forall x \in{\overline{\left\{y_{\varepsilon}\right\}}}^{\bar{q}} \text {. }
\end{aligned}
$$

In fact, Karapinar and Romaguera (2015) formulated a backward version (i.e. for right $q$ - $K$-completeness) of Theorem 5 . We presented here its forward analog.

The following theorem shows that Corollary 3 (see also Remark 9) gives a characterization of completeness of pseudo-quasimetric spaces.

Theorem 6 (a characterization of forward complete pseudo-quasimetric spaces) For $a$ pseudo-quasimetric space $(X, q)$ the following statements are equivalent.

(1) $(X, q)$ is forward-forward complete.

(2) For every proper, bounded from below and strictly-decreasing forward-lsc functional $\varphi: X \rightarrow \mathbb{R} \cup\{+\infty\}$, and for any $x_{0} \in \operatorname{dom} \varphi$, there is $x_{*} \in X$ such that for every $y_{*} \in S_{1, q}\left(x_{*}\right)=\left\{u \in X \mid \varphi(u)+q\left(x_{*}, u\right) \leq \varphi\left(x_{*}\right)\right\}$ one has

$$
\begin{aligned}
& \text { (i) } \varphi\left(y_{*}\right)+q\left(x_{0}, y_{*}\right) \leq \varphi\left(x_{0}\right) \text {; } \\
& \text { (ii) } \varphi(x)+q\left(y_{*}, x\right)>\varphi\left(y_{*}\right), \quad \forall x \in X \backslash \overline{\left\{y_{*}\right\}} \bar{q} \text {, and } \\
& \text { (iii) } \varphi\left(y_{*}\right) \leq \varphi(x), \quad \forall x \in \overline{\left\{y_{*}\right\}} \text {. }
\end{aligned}
$$

Proof (1) $\Rightarrow(2)$ By Proposition 3, the space $(X, q)$ is forward-distance-series-complete. Consequently, the hypotheses of Corollary 3 are fulfilled. Taking into account Remark 9, it follows that condition (iii) from (2) holds too.

$(2) \Rightarrow(1)$ Assume that $(X, q)$ is not forward-forward complete and show that there exists a function $\varphi$ satisfying the hypotheses from (2), but for which the conclusions (i)-(iii) fail.

By Proposition 3, $(X, q)$ is not forward-distance-series-complete so that there exists a sequence $\left\{x_{n}\right\}_{n \in \mathbb{N}_{0}}$ in $X$ with $\sum_{n=0}^{\infty} q\left(x_{n}, x_{n+1}\right)<\infty$, which has no forward limit. By Proposition 3 the sequence $\left\{x_{n}\right\}_{n \in \mathbb{N}_{0}}$ is forward-Cauchy, so that, by Theorem 1), it has no forward convergent subsequences.

We shall distinct two cases.

Case I. Suppose that

$$
\exists m, \forall k \geq m, \exists n_{k}>k, \rho\left(x_{k}, x_{n_{k}}\right)>0 .
$$

Then, for $n_{0}=m$ there exists $n_{1}>n_{0}$ such that $\rho\left(x_{n_{0}}, x_{n_{1}}\right)>0$. Taking $k=n_{1}$ it follows the existence of $n_{2}>n_{1}$ such that $\rho\left(x_{n_{1}}, x_{n_{2}}\right)>0$. Continuing in this manner we obtain a

\footnotetext{
18 A quasimetric in the sense of Karapinar et al. is a pseudo-quasimetric defined in Definition 2 satisfying that $x=y$ if and only if $q(x, y)=q(y, x)=0$.
} 
sequence $n_{0}<n_{1}<\ldots$ such that $\rho\left(x_{n_{k}}, x_{n_{k+1}}\right)>0$ for all $k \in \mathbb{N}_{0}$. Taking into account this subsequence and relabeling, we can suppose that

$$
\rho\left(x_{n}, x_{n+1}\right)>0, \quad \forall n \in \mathbb{N}_{0} .
$$

For

$$
s:=\sum_{k=1}^{\infty} q\left(x_{n}, x_{n+1}\right)<\infty .
$$

consider the function $\varphi: X \rightarrow \mathbb{R}$ defined by $\varphi(x)=2 s$ for all $x \in X \backslash\left\{x_{0}, x_{1}, x_{2}, \ldots\right\}, \varphi\left(x_{0}\right)=$ $s$ and $\varphi\left(x_{n+1}\right):=\varphi\left(x_{n}\right)-q\left(x_{n}, x_{n+1}\right)$ for all $n \geq 0$. Obviously, the sequence $\left\{x_{n}\right\}$ is strictly $\varphi$-decreasing, since $q\left(x_{n}, x_{n+1}\right)>0$ for all $n \in \mathbb{N}_{0}$. One obtains successively $\varphi\left(x_{1}\right)=\varphi\left(x_{0}\right)-q\left(x_{0}, x_{1}\right), \varphi\left(x_{2}\right)=\varphi\left(x_{1}\right)-q\left(x_{1}, x_{2}\right)=\varphi\left(x_{0}\right)-q\left(x_{0}, x_{1}\right)-q\left(x_{1}, x_{2}\right)$, and, by induction,

$$
\varphi\left(x_{n}\right)=\varphi\left(x_{0}\right)-\sum_{i=0}^{n-1} q\left(x_{i}, x_{i+1}\right) .
$$

Taking into account that $\varphi\left(x_{0}\right)=s$, it follows

$$
\varphi\left(x_{n}\right)=\sum_{i=n}^{\infty} q\left(x_{i}, x_{i+1}\right)>0,
$$

which shows that the functional $\varphi$ is bounded from below by 0 . Since any strictly $\varphi$-decreasing sequence must be a subsequence of $\left\{x_{n}\right\}$, it follows that there are no strictly $\varphi$-decreasing forward convergent sequences, implying that the functional $\varphi$ is strictly $\varphi$-decreasing forwardlsc. By hypothesis, there exists $x_{*} \in X$ satisfying the conditions (i)-(iii). Condition (i) implies $q\left(x_{0}, x_{*}\right) \leq \varphi\left(x_{0}\right)-\varphi\left(x_{*}\right)$, hence $\varphi\left(x_{*}\right) \leq \varphi\left(x_{0}\right)=s$, so that $x_{*}=x_{m}$ for some $m \in \mathbb{N}_{0}$. Condition (ii) for $x=x_{m+1}$ yields

$$
\varphi\left(x_{m+1}\right)>\varphi\left(x_{m}\right)-q\left(x_{m}, x_{m+1}\right),
$$

in contradiction to the definition of $\varphi$.

Case II. Suppose that (3.9) does not hold, that is

$$
\forall m, \exists k \geq m, \text { s.t. } \forall n>k, \rho\left(x_{k}, x_{n}\right)=0 .
$$

For $m=0$ let $k=n_{0} \geq 0$ be such that $\rho\left(x_{n_{0}}, x_{n}\right)=0$ for all $n>n_{0}$. Now, for $m=1+n_{0}$ let $n_{1}>n_{0}$ be such that $\rho\left(x_{n_{1}}, x_{n}\right)=0$ for all $n>n_{1}$. It follows $\rho\left(x_{n_{0}}, x_{n_{1}}\right)=0$.

Continuing in this manner we obtain a sequence $n_{0}<n_{1}<\ldots$ such that $\rho\left(x_{n_{k}}, x_{n_{k+1}}\right)=$ 0 for all $k \in \mathbb{N}_{0}$. Relabeling, we can suppose that the sequence $\left(x_{n}\right)$ satisfies

$$
\rho\left(x_{n}, x_{n+1}\right)=0, \forall n \in \mathbb{N}_{0} .
$$

Let $B:=\left\{x_{n}: n \in \mathbb{N}_{0}\right\}$, and define the function $\varphi: X \rightarrow \mathbb{R}$ by

$$
\varphi(x)= \begin{cases}\frac{1}{2^{n}} & \text { if } x=x_{n} \text { for some } n \in \mathbb{N}_{0}, \\ 2 & \text { for } x \in X \backslash B .\end{cases}
$$

Again, any strictly $\varphi$-decreasing sequence must be a subsequence of $\left\{x_{n}\right\}$, implying that there are no strictly $\varphi$-decreasing forward convergent sequences, so that the functional $\varphi$ is strictly $\varphi$-decreasing forward-1sc. 
Suppose that there exists $x_{*} \in X$ satisfying the conditions (i)-(iii). By (i), $\varphi\left(x_{*}\right) \leq \varphi\left(x_{0}\right)=$ 1 , so that $y=x_{m} \in B$ for some $m \in \mathbb{N}_{0}$.

If (3.13) holds, then, by the triangle inequality,

$$
\rho\left(x_{m}, x_{m+k}\right) \leq \sum_{i=1}^{k} \rho\left(x_{m+i-1}, x_{m+i}\right)=0,
$$

i.e. $x_{n} \in{\overline{\left\{x_{m}\right\}}}^{\bar{q}}$, for all $n \geq m$. By (iii),

$$
\varphi\left(x_{m}\right) \leq \varphi\left(x_{n}\right)=\frac{1}{2^{n}}, \quad \forall n \geq m,
$$

implying $\varphi\left(x_{m}\right)=0$, a value not taken by $\varphi$.

Remark 12 The function considered in Case II in the above proof is inspired by one considered in Karapinar and Romaguera (2015, Theorem 2). Note that the authors of Karapinar and Romaguera (2015) did not consider this case (i.e. the possibility that $q\left(x_{n}, x_{n+1}\right)=0$ for all $n$ ) in their proof.

The following example shows that condition (iii) in Theorem 6 is essential for the completeness of the pseudo-quasimetric space $(X, q)$.

Example 8 Let $x_{n}=-n, n \in \mathbb{N}_{0}$, and $X=\left\{x_{n}: n \in \mathbb{N}_{0}\right\}$ with the metric $q\left(x_{n}, x_{m}\right)=$ $\left(x_{m}-x_{n}\right)^{+}=(-m+n)^{+}=n-m$ if $n>m$ and $=0$ if $n \leq m$. Then $q\left(x_{n}, x_{n+1}\right)=0$ for all $n \in \mathbb{N}_{0}$ and the space $(X, q)$ is not forward-complete (see Remark 6). Let $\varphi: X \rightarrow[0, \infty)$ be an arbitrary function. For $x_{*}=x_{0}$,

$$
\varphi\left(x_{*}\right)=\varphi\left(x_{0}\right) \leq \varphi\left(x_{0}\right)=\varphi\left(x_{0}\right)+q\left(x_{*}, x_{0}\right) .
$$

Since $q\left(x_{0}, x_{n}\right)=0$ for all $n \in \mathbb{N}_{0}$ the condition

$$
\varphi\left(x_{0}\right)<\varphi\left(x_{n}\right)+q\left(x_{0}, x_{n}\right), \forall n \in \mathbb{N}_{0}: q\left(x_{0}, x_{n}\right)>0
$$

is trivially satisfied.

\section{Behavioral applications: when completeness is equivalent to the existence of traps}

Soubeyran $(2009,2010,2016)$ showed that, quite surprisingly, Ekeland's variational principle offers a prototype of the variational rationality model. In this simple but very important benchmark case, we have:

- The space $(X, q)$ is a quasimetric or a pseudo-quasimetric space. It refers to a space of actions (doings), havings or beings, where $q: X \times X \rightarrow \mathbb{R}$ is a quasimetric or a pseudo-quasimetric.

- The scalar function $g: X \rightarrow \mathbb{R}$ is a "to be increased payoff", some profit or satisfaction level, $\bar{g}=\sup _{x \in X} g(x)<+\infty$ is the highest payoff the agent can expect to realize (a maximum seen as an aspiration level), while $\varphi(x)=\bar{g}-g(x) \geq 0$ refers to his "to be decreased" residual dissatisfaction to fail to reach $\bar{g}$.

- Advantages to change are $A(x, y)=g(y)-g(x)=\varphi(x)-\varphi(y)$.

- Inconveniences to change are $I(x, y)=C(x, y)-C(x, x) \geq 0$, where $C(x, y) \geq 0$ refers to costs to be able to change and $C(x, x) \geq 0$ represents costs to be able to stay. 
- The worthwhile-to-change ratio $\lambda$ which defines how, each period, it is worthwhile to change, is constant all along the transition.

Soubeyran $(2009,2010,2016)$ has shown, in great details, in which circumstances inconveniences to change can be modeled as pseudo-quasi-metrics $q(x, y)=I(x, y)=$ $C(x, y)-C(x, x) \geq 0$. In this case, inconveniences to change can be zero even when $y \neq x$. This means that costs to be able to change $C(x, y)$ can be equal to costs to be able to stay $C(x, x)$ for some change $x \curvearrowright y \neq x$. Be cautious, these costs to be able to do are very different from traditional costs to do (execution costs).

Then, in the current period $n+1$, a change which moves from repeating the last action $x=x_{n} \in X$ to perform the current action $y=x_{n+1} \in X$ is worthwhile if $A(x, y) \geq \lambda I(x, y)$, where the highest is $\lambda>0$, the more it is worthwhile to change. A worthwhile change $x \curvearrowright y$ satisfies $\varphi(x)-\varphi(y) \geq \lambda q(x, y)$. Given $\varphi(),$.$x and \lambda$, the (VR) approach defines, each current period, the worthwhile to change set, $S_{\lambda, q}(x)=\{y \in X \mid \varphi(x)-\varphi(y) \geq \lambda q(x, y)\}$ and the related worthwhile stay and change dynamic $x_{n+1} \in S_{\lambda, q}\left(x_{n}\right)$. A current stay is such that $x_{n+1}=x_{n}$, while a current change is such that $x_{n+1} \neq x_{n}$. A variational trap $x_{*} \in X$ is worthwhile to approach, i.e., $x_{n+1} \in S_{\lambda, q}\left(x_{n}\right)$ and worthwhile to reach, i.e., $x_{*} \in S_{\lambda, q}\left(x_{n}\right)$ for all $n \in \mathbb{N}$ but not worthwhile to leave, that is $S_{\lambda, q}\left(x_{*}\right)=\left\{x_{*}\right\}$. Then, it is easy to see that Ekeland's variational principle gives sufficient conditions for the existence of variational traps, starting from any initial action.

Our paper gives sufficient conditions for the existence of variational traps in the case where inconveniences to change are pseudo-quasimetrics. Furthermore, our paper shows that completeness of the pseudo-quasimetric space $X$ is equivalent to the existence of variational traps. It means that in such pseudo-quasimetric spaces, the fact that every worthwhile stay and change dynamic ends in some trap is equivalent to completeness. Then, in such complete spaces, no worthwhile stay and change dynamic can wander. Then, each period, if an agent prefers to change from his previous position to a new one, because such change is worthwhile, sooner or later, he will end in a trap where it is not worthwhile to change. This is a very nice result in Behavioral Sciences.

\section{Conclusions}

In this paper, we establish new versions of Ekeland's variational principle for strictdecreasingly forward- (resp. backward-)lsc functions in possible incomplete pseudo-quasimetric spaces; in particular, in $\mathbb{R}$ with $q(x, y)=y-x$ if $y \geq x$ and 0 otherwise. It is important to emphasize that the motivation of this research comes from applications in behavioral sciences.

We further extend the characterization of the completeness of $T_{1}$-quasimetric (resp. quasimetric) spaces in Cobzaş (2011), (resp. Karapinar and Romaguera 2015).

A natural question arise: why matters the use of pseudo-quasimetrics instead of quasimetrics in the statements of variational principles? That is, why the existence of $y$ different from $x$ such that $q(x, y)=0$ does matter so much?

The motivation comes from its interest in applications to Behavioral Sciences, based on the VR approach, where passing from quasidistances to pseudo-quasidistances as a model for inconveniences to change is essential, as explained below.

In this case a change from $x$ to $y, y$ different from $x$, can generate no inconveniences, that is $I(x, y)=C(x, y)-C(x, x)=q(x, y)=0$, because costs of being able to change $C(x, y)$ equal costs of being able to stay, $C(x, x)$. In this case a worthwhile change from $x$ to 
$y$ is such that $g(y)-g(x) \geq h q(x, y)=0$, that is such that $g(y) \geq g(x)$. Then, in this case, an improving change, and even more a strictly increasing change, becomes worthwhile.

This is also why our assumptions on strictly $\varphi$-decreasing sequences and strictdecreasingly forward-lsc functions are natural and matter much.

Ekeland's variational principle has been investigated in different setting; (see, e.g., Ansari 2014; Bao et al. 2016). In Al-Homidan et al. (2008) the authors introduced the notion of $Q$-distance in quasi-metric spaces and established an equilibrium version of the Ekelandtype variational principle. Our further research will examize whether our results could be formulated in terms of a $Q$ distance.

Acknowledgements The contribution of the first author was completed while visiting Aix-Marseille University with a GREQAM-AMSE grant.

\section{References}

Al-Homidan, S., Ansari, Q. H., \& Yao, J.-C. (2008). Some generalizations of Ekeland-type variational principle with applications to equilibrium problems and fixed point theory. Nonlinear Analysis, Theory, Methods and Applications, 69(1), 126-139.

Ansari, Q. H. (2007). Vectorial form of Ekeland-type variational principle with applications to vector equilibrium problems and fixed point theory. Journal of Mathematical Analysis and Applications, 334(2), 561-575.

Ansari, Q. H. (2014). Ekeland's variational principle and its extensions with applications. In S. Almezel, Q. H. Ansari, \& M. A. Khamsi (Eds.), Topics in fixed point theory (pp. 65-100). Heidelberg: Springer.

Aydi, H., Karapınar, E., \& Vetro, C. (2015). On Ekeland's variational principle in partial metric spaces. Applied Mathematics and Information Sciences, 9, 257-262.

Bao, T. Q. (2015). Modelling, computation and optimization in information systems and management sciences. In L. T. Hoai An, P. D. Tao, \& N. N. Thanh (Eds.), Vectorial Ekeland variational principles: A hybrid approach (pp. 513-525). Heidelberg: Springer.

Bao, T. Q., \& Théra, M. A. (2015). On extended versions of Dancs-Hegedüs-Medvegyev's fixed-point theorem. Optimization. doi:10.1080/02331934.2015.1113533.

Bao, T. Q., Mordukhovich, B. S., \& Soubeyran, A. (2015a). Variational analysis in psychological modeling. Journal of Optimization Theory and Applications, 164, 290-315.

Bao, T. Q., Mordukhovich, B. S., \& Soubeyran, A. (2015b). Fixed points and variational principles with applications to capability theory of wellbeing via variational rationality. Set-Valued Variational Analysis, 23, 375-398.

Bao, T. Q., Mordukhovich, B. S., \& Soubeyran, A. (2015c). Minimal points, variational principles, and variable preferences in set optimization. Journal of Nonlinear Convex Analysis, 16, 1511-1537.

Bao, T. Q., Khanh, P. Q., \& Soubeyran, A. (2016). Variational principles with generalized distances and the modelization of organizational change. Optimization, 65, 2049-2066.

Cobzaş, S. (2011). Completeness in quasi-metric spaces and Ekeland variational principle. Topology and its Applications, 158, 1073-1084.

Cobzaş, S. (2013). Functional analysis in asymmetric normed spaces. Frontiers in mathematics. Basel: Birkhäuser/Springer Basel AG.

Cobzaş, S. (2016). Fixed points and completeness in metric and in generalized metric spaces, pp. 1-71. arXiv: $1508.05173 \mathrm{v} 4$

Dancs, S., Hegedüs, M., \& Medvegyev, P. (1983). A general ordering and fixed-point principle in complete metric space. Acta Scientiarum Mathematicarum (Szeged), 46, 381-388.

Ekeland, I. (1972). Sur les problèmes variationnels. Comptes rendus de l'Académie des Sciences Sér. A-B, 275, 1057-1059.

Ekeland, I., \& Turnbull, T. (1983). Infinite-dimensional optimization and convexity. Chicago lectures in mathematics. Chicago, IL: University of Chicago Press.

Filip, A.-D. (2011). Fixed point theory in Kasahara spaces. Babeş-Bolyai University, Thesis (Ph.D.)-BabeşBolyai University, Cluj-Napoca, Romania.

Gierz, G., Hofmann, K. H., Keimel, K., Lawson, J. D., Mislove, M., \& Scott, D. S. (2003). Continuous lattices and domains. Encyclopedia of mathematics and its applications. Cambridge: Cambridge University Press. 
Goubault-Larrecq, J. (2013). Non-Hausdorff topology and domain theory: Selected topics in point-set topology. New Mathematical Monographs, Vol. 22. Cambridge: Cambridge University Press.

Karapinar, E., \& Romaguera, S. (2015). On the weak form of Ekeland's variational principle in quasi-metric spaces. Topology and its Applications, 184, 54-60.

Kelley, J. L. (1975). General topology, 2nd ed. Graduate Texts in Mathematics, Vol. 27. New York: Springer.

Kelly, J. C. (1963). Proceedings London Mathematical Society. Bitopological Spaces, 13, 71-89.

Khanh, P. Q., \& Quy, D. N. (2013). Versions of Ekeland's variational principle involving set perturbations. Journal of Global Optimization, 57, 951-968.

Kirk, W. A., \& Saliga, L. M. (2001). The Brézis-Browder order principle and extensions of Caristi's theorem. Nonlinear Analysis, 47, 2765-2778.

Lin, L.-J., Wang, S.-Y., \& Ansari, Q. H. (2011). Critical point theorems and Ekeland type variational principle with applications. Fixed Point Theory and Applications, Art. ID, 914624, 1-21.

Megginson, R. E. (1998). An introduction to Banach space theory. Graduate Texts in Mathematics, Vol. 183. New York: Springer.

Reilly, I. L., \& Subrahmanyam, P. V. (1982). Cauchy sequences in quasipseudometric spaces. Monatshefte für Mathematik, 93, 127-140.

Soubeyran, A. (2009). Variational rationality, a theory of individual stability and change: Worthwhile and ambidextry behaviors. Preprint at GREQAM, Aix-Marseille University.

Soubeyran, A. (2010). Variational rationality and the "unsatisfied man" : Routines and the course pursuit between aspirations, beliefs. Preprint at GREQAM. Aix-Marseille University.

Soubeyran, A. (2016). Variational rationality. Part 1. Worthwhile stay and change approach-avoidance human dynamics ending in traps. Preprint at GREQAM, Aix-Marseille University.

Sullivan, F. (1981). A characterization of complete metric spaces. Proceedings of the American Mathematical Society, 83, 345-346.

Ume, J. S. (2002). A minimization theorem in quasi-metric spaces and its applications. International Journal of Mathematics and Mathematical Sciences, 31, 443-447.

Wilson, W. A. (1931a). On quasi-metric spaces. American Journal of Mathematics, 53, 675-684.

Wilson, W. A. (1931b). On semi-metric spaces. American Journal of Mathematics, 53, 361-373. 